Interfaces and Free Boundaries 14 (2012), 401-430

DOI $10.4171 / \mathrm{IFB} / 286$

\title{
Topology optimization methods with gradient-free perimeter approximation
}

\author{
SAMUEL AMSTUTZ \\ Laboratoire de Mathématiques d'Avignon, Faculté des Sciences, 33 rue Louis Pasteur, 84000 \\ Avignon, France \\ E-mail: samuel.amstutz@univ-avignon.fr \\ Nicolas VAN GOETHEM \\ Universidade de Lisboa, Faculdade de Ciências, Departamento de Matemática, Centro de \\ Matemática e Aplicações Fundamentais, Av. Prof. Gama Pinto 2, 1649-003 Lisboa, Portugal \\ E-mail:vangoeth@ptmat.fc.ul.pt
}

[Received 18 January 2012 and in revised form 28 June 2012]

\begin{abstract}
In this paper we introduce a family of smooth perimeter approximating functionals designed to be incorporated within topology optimization algorithms. The required mathematical properties, namely the $\Gamma$-convergence and the compactness of sequences of minimizers, are first established. Then we propose several methods for the solution of topology optimization problems with perimeter penalization showing different features. We conclude by some numerical illustrations in the contexts of least square problems and compliance minimization.
\end{abstract}

2010 Mathematics Subject Classification: Primary 49Q10, 49Q20; Secondary 35J05, 35J25.

Keywords: Topology optimization, perimeter, $\Gamma$-convergence, homogenization

\section{Introduction}

In several areas of applied sciences, models where the perimeter of an unknown set plays a crucial role may be considered. Such problems encompass multiphase problems where the interface between two liquid phases is assumed to minimize a free energy while keeping its area bounded [24, 32], or image segmentation models with Mumford-Shah [7] type functionals. A related problem is that of minimal partitions $[12,14,19]$. Specifically, consider a bounded domain $\Omega$ of $\mathbb{R}^{2}$, a number $m \in \mathbb{N}$, functions $g_{1}, \ldots, g_{m} \in L^{1}(\Omega)$, and a parameter $\alpha>0$. A model problem of minimal partition reads

$$
\min _{\Omega_{1}, \ldots, \Omega_{m}} \sum_{i=1}^{m}\left[\int_{\Omega_{i}} g_{i}(x) d x+\frac{\alpha}{2} \operatorname{Per}\left(\Omega_{i}\right)\right],
$$

where the minimum is searched among all partitions $\left(\Omega_{1}, \ldots, \Omega_{m}\right)$ of $\Omega$ by subsets of finite perimeter and were $\operatorname{Per}\left(\Omega_{i}\right)$ is the relative perimeter of $\Omega_{i}$ in $\Omega$. Another important field where the perimeter comes into play is the optimal design of shapes [6], such as load bearing structures or electromagnetic devices, where it aims at rendering the problem well-posed in the sense of the existence of optimal domains. Indeed, it is known that a perimeter constraint provides in many shape optimization problems an extra compactness that leads to the existence of a domain solution, while on the contrary homogenization would occur if the perimeter constraint was removed. 
However it is known that a major difficulty of standard perimeter penalization is that the sensitivity of the perimeter to topology changes is of lower order compared to usual cost functionals, like volume integrals (see, e.g., [9, 23, 31] for the topological sensitivity of various functionals) and thus prohibits a successful numerical solution. In this paper we propose a regularization of the perimeter that overcomes this drawback and show simple applications in topology optimization and source identification. Since we believe that applications of our method could be useful in other areas of applied sciences, a brief overview of the physical motivation of our approach is first proposed.

The Ericksen-Timoshenko bar [30] was designed as an alternative to strain-gradient models to simulate microstructures of finite scale $\varepsilon$, where an energy functional

$$
G_{\varepsilon}(u, v)=\int_{0}^{1}\left(\frac{\varepsilon^{2}}{2}\left(v^{\prime}\right)^{2}+\frac{1}{2}(u-v)^{2}\right) d x
$$

depending on two variables $u$, the longitudinal strain, and $v$, an internal variable assumed to measure all deviations from $1 D$ deformations, is minimized in $(u, v)$. Seeking a minimum in the second variable amounts to finding $v_{\varepsilon}$ solution of the Euler-Lagrange equation $-\varepsilon^{2} v_{\varepsilon}^{\prime \prime}+v_{\varepsilon}=u$ with $v_{\varepsilon}^{\prime}(0)=v_{\varepsilon}^{\prime}(1)=0$. Hence the problem can be restated as

$$
u_{\varepsilon} \in \operatorname{argmin} F_{\varepsilon}(u):=\frac{1}{\varepsilon} G_{\varepsilon}\left(u, v_{\varepsilon}\right)=\frac{\alpha}{2 \varepsilon}\left\langle u-v_{\varepsilon}, u\right\rangle,
$$

where the brackets denote the $L^{2}$ scalar product. Moreover, it is observed that $v_{\varepsilon}$ also minimizes $\tilde{G}_{\varepsilon}(u, v):=\frac{1}{\varepsilon} G_{\varepsilon}(u, v)+\frac{1}{2 \varepsilon}\langle u, 1-u\rangle$ which, in two papers of Gurtin and Fried [21,22], is identified with the free energy of (a particular choice of ${ }^{\ddagger}$ ) some thermally induced phase transition models where $u$ stands for the scaled temperature variation and $v$ represents a scalar "order parameter". In [22], the authors consider a dimensional analysis where $\varepsilon$ is allowed to tend to 0 .

In the present paper, the problem is considered for an arbitrary space dimension $N$. Our approximating functional is based on the minimization in the second variable of

$$
\tilde{G}_{\varepsilon}(u, v)=\frac{1}{2 \varepsilon} \int_{\Omega}\left(u(1-u)+(u-v)^{2}\right) d x+\frac{\varepsilon}{2} \int_{\Omega}|\nabla v|^{2} d x,
$$

thereby involving for each selected $\varepsilon$ the solution $v_{\varepsilon}$ of the following PDE:

$$
\left\{\begin{array}{ll}
-\varepsilon^{2} \Delta v_{\varepsilon}+v_{\varepsilon}=u & \text { in } \Omega \\
\partial_{n} v_{\varepsilon}=0 & \text { on } \partial \Omega .
\end{array} .\right.
$$

Specifically, in this paper we show that the function

$$
\tilde{F}_{\varepsilon}(u):=\inf _{v \in H^{1}(\Omega)} \tilde{G}_{\varepsilon}(u, v)=\tilde{G}_{\varepsilon}\left(u, v_{\varepsilon}\right)=\frac{1}{2 \varepsilon}\left\langle 1-v_{\varepsilon}, u\right\rangle
$$

for $u \in L^{\infty}(\Omega,[0,1])$ is the relaxation with respect to the weak-* topology of the functional defined by $F_{\varepsilon}(u):=\frac{1}{2 \varepsilon}\left\langle u-v_{\varepsilon}, u\right\rangle$ if $u \in L^{\infty}(\Omega,\{0,1\})$, and $+\infty$ otherwise in $L^{\infty}(\Omega,[0,1])$. Then, we prove that $\alpha^{-1} \tilde{F}_{\varepsilon}(u)$ converges as $\varepsilon \rightarrow 0$ in a suitable sense and for a particular value of $\alpha$ independent of $N$ to the perimeter $\operatorname{Per}(A)$ of $A$ as soon as $u$ is the characteristic function of some

* In particular a model with dissipationless kinetics and vanishing specific heat. 
subset $A$ of $\Omega$, and to $+\infty$ otherwise. As a consequence we can address topology optimization problems where the perimeter is approximated by $\alpha^{-1} \tilde{F}_{\varepsilon}\left(\chi_{A}\right)$ with $A$ in some admissible class of shapes. The behavior of the functional $\tilde{F}_{\varepsilon}$ with respect to optimization is enhanced by the fact that it is by construction weakly-* continuous on $L^{\infty}(\Omega,[0,1])$ and obviously free of any gradient term. Let us emphasize that, while the addition of the perimeter in several shape and topology optimization problems is by now quite standard, it is usually done in an ad-hoc manner to penalize an optimization algorithm, see [15] and the references therein. To our knowledge a proper mathematical justification is still missing and we believe that the contribution of this paper is also to propose a theoretical response to this important issue.

Since we intend to analyze the convergence of minimizers as $\varepsilon \rightarrow 0$, a general notion of convergence of functionals, namely the $\Gamma$-convergence $[18,20]$, must be considered. In this setting, the Modica-Mortola approach to approximate the perimeter is well-known (the reference papers are [27-29]). In image segmentation [7] or fracture mechanics [5, 17, 33], the length of the jump set of the unknown $u$ is added to quadratic terms integrated over the smooth regions, whose joint regularization is provided by the celebrated Ambrosio-Tortorelli functional [8]. Let us emphasize that, as they involve a gradient term $\|\nabla u\|_{L^{2}}^{2}$, the two aforementioned functionals present several drawbacks in order to approximate optimal solutions in topology optimization. First, they are defined for $H^{1}$ functions and not for characteristic functions, hence they would require to extend the cost function to the intermediate values, typically by a relaxation which is not always doable. Second, they are not compatible with a discretization of $u$ by piecewise constant finite elements, which are yet the most frequently used in topology optimization. Third, as observed in [16] where the Modica-Mortola functional was combined with a phase field approach, the Laplacian of $u$ appearing when differentiating the gradient term leads to numerical instabilities in the optimization process, requiring the use of rather sophisticated semi-implicit schemes.

In a previous paper [10] the pointwise convergence of a variant of $F_{\varepsilon}\left(\chi_{A}\right)$ to $\operatorname{Per}(A)$ for $A$ with suitable regularity has been studied. Moreover, the topological sensitivity (or derivative) of the approximating functionals has been explicitly computed. With our approximating functionals $\tilde{F}_{\varepsilon}$ or $F_{\varepsilon}$ we are able to nucleate holes, in particular, we can compute the corresponding topological derivatives at the only additional cost of computing an adjoint state solution to a well-posed elliptic PDE similar to (1.3) with appropriate right hand-side. Moreover, if topology optimization is intended without using the concept of topological derivative, our formulation allows one to relax the cost function, yielding minimizing sequences showing intermediate "homogenized" values, but nevertheless converging to a characteristic function. This is a simple consequence of the fact that $\tilde{F}_{\varepsilon}(u) \rightarrow+\infty$ as soon as $u$ takes values outside $\{0,1\}$. In this respect, it can be seen as a particular way to penalize intermediate densities in homogenization methods, which is usually done by heuristic techniques [2]. From a numerical point of view another direct benefit of our approach is that some special but important topology optimization problems can be explicitly written as multiple infima, which are efficiently handled by alternating directions algorithms.

It is rather remarkable that $\tilde{F}_{\varepsilon}(u)$ seems not only to be arbitrarily proposed to get better numerical algorithms, but also has an intrinsic meaning in terms of physical modeling, i.e., as a free-energy type functional depending on a small parameter and where $v$ is interpreted as a slow internal variable which tracks the fast variable $u$. Our approach can therefore be a tool to study limit models as $\varepsilon \rightarrow 0$. In fracture mechanics one may think for instance of fracture models approximated by damage models, where the damage variable is the scalar $v, \varepsilon$ is the "thickness" of the crack, and $u$ the displacement field, while the cost function is a Griffith-type energy [5, 17]. Coming back 
to our first motivation example, the Eriksen-Timoshenko bar, there is an interest to replace straingradient models by models with internal variables and free energy functionals reading as our $F_{\varepsilon}$. We believe that several other problems in physics where the perimeter enters the model could also find appropriate interpretations and/or extension in the light of our functional.

The rest of the paper is organized as follows. The basic properties of the functionals $F_{\varepsilon}$ and $\tilde{F}_{\varepsilon}$ are studied in Section 2. The $\Gamma$-convergence is proved in Section 3, using as essential ingredient a result from [32]. Our main results concerning the solution of topology optimization problems are established in Section 4. Sections 5 through 7 are devoted to numerical applications. Concluding remarks are given in Section 8.

\section{Description of the approximating functionals}

Let $\Omega$ be a bounded domain of $\mathbb{R}^{N}$ with Lipschitz boundary. For all $u \in L^{2}(\Omega)$ we define

$$
F_{\varepsilon}(u):=\inf _{v \in H^{1}(\Omega)}\left\{\frac{\varepsilon}{2}\|\nabla v\|_{L^{2}(\Omega)}^{2}+\frac{1}{2 \varepsilon}\|v-u\|_{L^{2}(\Omega)}^{2}\right\}
$$

i.e., $F_{\varepsilon}$ is equal to $\varepsilon / 2$ times the Moreau-Yosida regularization with constant $1 / \varepsilon^{2}$ of the function $v \in H^{1}(\Omega) \mapsto\|\nabla v\|_{L^{2}(\Omega)}^{2}$. We next introduce the functional $\tilde{F}_{\varepsilon}$ given by

$$
\tilde{F}_{\varepsilon}(u)=F_{\varepsilon}(u)+\frac{1}{2 \varepsilon}\langle u, 1-u\rangle
$$

or equivalently after simplification

$$
\tilde{F}_{\varepsilon}(u):=\inf _{v \in H^{1}(\Omega)}\left\{\frac{\varepsilon}{2}\|\nabla v\|_{L^{2}(\Omega)}^{2}+\frac{1}{2 \varepsilon}\left(\|v\|_{L^{2}(\Omega)}^{2}+\langle u, 1-2 v\rangle\right)\right\} .
$$

Throughout we use the notation $\langle u, v\rangle:=\int_{\Omega} u v d x$ for every pair of functions $u$, $v$ having suitable regularity. In Proposition 2.4 we shall show that the functional $\tilde{F}_{\varepsilon}$ restricted to the space $L^{\infty}(\Omega,[0,1])$ is the relaxation with respect to the weak-* topology of the functional defined by $F_{\varepsilon}$ if $u \in L^{\infty}(\Omega,\{0,1\})$, and $+\infty$ otherwise in $L^{\infty}(\Omega,[0,1])$. Before that, we give practical expressions of these functionals based on the Euler-Lagrange equations of the corresponding minimization problems.

Proposition 2.1 Let $u \in L^{2}(\Omega)$ be given and $v_{\varepsilon} \in H^{1}(\Omega)$ be the (weak) solution of

$$
\begin{cases}-\varepsilon^{2} \Delta v_{\varepsilon}+v_{\varepsilon}=u & \text { in } \Omega \\ \partial_{n} v_{\varepsilon}=0 & \text { on } \partial \Omega\end{cases}
$$

Then we have

$$
\begin{aligned}
& F_{\varepsilon}(u)=\frac{1}{2 \varepsilon}\left\langle u-v_{\varepsilon}, u\right\rangle, \\
& \tilde{F}_{\varepsilon}(u)=\frac{1}{2 \varepsilon}\left\langle 1-v_{\varepsilon}, u\right\rangle .
\end{aligned}
$$

Moreover, $\tilde{F}_{\varepsilon}(u)$ is differentiable with respect to $\varepsilon$ with derivative

$$
\frac{d}{d \varepsilon} \tilde{F}_{\varepsilon}(u)=\frac{1}{2 \varepsilon^{2}}\left[3\left\langle u, v_{\varepsilon}\right\rangle-2\left\|v_{\varepsilon}\right\|_{L^{2}(\Omega)}^{2}-\langle 1, u\rangle\right] .
$$


Proof. The Euler-Lagrange equations of the minimization problems (2.1) and (2.3) are identical and read for the solution $v_{\varepsilon}$

$$
\varepsilon^{2}\left\langle\nabla v_{\varepsilon}, \nabla \varphi\right\rangle+\left\langle v_{\varepsilon}, \varphi\right\rangle=\langle u, \varphi\rangle \quad \forall \varphi \in H^{1}(\Omega),
$$

which is the weak formulation of (2.4). It holds in particular

$$
\varepsilon^{2}\left\|\nabla v_{\varepsilon}\right\|_{L^{2}(\Omega)}^{2}+\left\|v_{\varepsilon}\right\|_{L^{2}(\Omega)}^{2}=\left\langle v_{\varepsilon}, u\right\rangle .
$$

Plugging (2.9) into (2.1) and (2.3) entails (2.5) and (2.6). Let $\dot{v}_{\varepsilon}$ denote the derivative of $v_{\varepsilon}$ with respect to $\varepsilon$, whose existence is easily deduced from the implicit function theorem. Differentiating (2.6) by the chain rule yields

$$
\frac{d}{d \varepsilon} \tilde{F}_{\varepsilon}(u)=-\frac{1}{2 \varepsilon^{2}}\left\langle 1-v_{\varepsilon}, u\right\rangle-\frac{1}{2 \varepsilon}\left\langle\dot{v}_{\varepsilon}, u\right\rangle .
$$

Using (2.8) we obtain

$$
\frac{d}{d \varepsilon} \tilde{F}_{\varepsilon}(u)=-\frac{1}{2 \varepsilon^{2}}\left\langle 1-v_{\varepsilon}, u\right\rangle-\frac{1}{2 \varepsilon}\left[\varepsilon^{2}\left\langle\nabla v_{\varepsilon}, \nabla \dot{v}_{\varepsilon}\right\rangle+\left\langle v_{\varepsilon}, \dot{v}_{\varepsilon}\right\rangle\right] .
$$

Now differentiating (2.8) provides

$$
2 \varepsilon\left\langle\nabla v_{\varepsilon}, \nabla \varphi\right\rangle+\varepsilon^{2}\left\langle\nabla \dot{v}_{\varepsilon}, \nabla \varphi\right\rangle+\left\langle\dot{v}_{\varepsilon}, \varphi\right\rangle=0 \quad \forall \varphi \in H^{1}(\Omega) .
$$

Choosing $\varphi=v_{\varepsilon}$ yields

$$
\varepsilon^{2}\left\langle\nabla \dot{v}_{\varepsilon}, \nabla v_{\varepsilon}\right\rangle+\left\langle\dot{v}_{\varepsilon}, v_{\varepsilon}\right\rangle=-2 \varepsilon\left\|\nabla v_{\varepsilon}\right\|_{L^{2}(\Omega)}^{2} .
$$

It follows from (2.10) that

$$
\frac{d}{d \varepsilon} \tilde{F}_{\varepsilon}(u)=-\frac{1}{2 \varepsilon^{2}}\left\langle 1-v_{\varepsilon}, u\right\rangle+\left\|\nabla v_{\varepsilon}\right\|_{L^{2}(\Omega)}^{2} .
$$

Using (2.9) and rearranging yields (2.7).

REMARK 2.2 Conversely, there is a natural way to retrieve (2.1) and (2.3) from (2.5) and (2.6) by Legendre-Fenchel transform. Indeed, the function $Q_{\varepsilon}: u \in H^{1}(\Omega)^{\prime} \mapsto\left\langle u, v_{\varepsilon}\right\rangle_{H^{1}(\Omega)^{\prime}, H^{1}(\Omega)}$ with $v_{\varepsilon} \in H^{1}(\Omega)$ solution to (2.8) is convex and continuous. Hence $Q_{\varepsilon}$ is equal to its biconjugate $Q_{\varepsilon}^{* *}$ (see e.g. [13]). A short calculation provides, for all $v \in H^{1}(\Omega)$,

$$
Q_{\varepsilon}^{*}(2 v)=\varepsilon^{2}\|\nabla v\|_{L^{2}(\Omega)}^{2}+\|v\|_{L^{2}(\Omega)}^{2} .
$$

This entails for all $u \in L^{2}(\Omega)$

$$
\left\langle u, v_{\varepsilon}\right\rangle=Q_{\varepsilon}(u)=Q_{\varepsilon}^{* *}(u)=-\inf _{v \in H^{1}(\Omega)} \varepsilon^{2}\|\nabla v\|_{L^{2}(\Omega)}^{2}+\|v\|_{L^{2}(\Omega)}^{2}-2\langle u, v\rangle,
$$

from which we straightforwardly derive (2.1) and (2.3). 
It is well-known that the set $L^{\infty}(\Omega,[0,1])$ is the convex hull of $L^{\infty}(\Omega,\{0,1\})$. Let us now prove the relaxation result for $F_{\varepsilon}$. Setting for $u \in L^{\infty}(\Omega,[0,1])$

$$
\bar{F}_{\varepsilon}(u):= \begin{cases}F_{\varepsilon}(u) & \text { if } u \in L^{\infty}(\Omega,\{0,1\}) \\ +\infty & \text { otherwise in } L^{\infty}(\Omega,[0,1])\end{cases}
$$

we will show that $\tilde{F}_{\varepsilon}$ as defined by (2.3) is the lower semicontinuous envelope (or relaxation) of $\bar{F}_{\varepsilon}$ with respect to the weak-* topology, that is,

$$
\tilde{F}_{\varepsilon}(u)=\inf \left\{\liminf _{n \rightarrow \infty} \bar{F}_{\varepsilon}\left(u_{n}\right): u_{n} \in L^{\infty}(\Omega,[0,1]), u_{n} \stackrel{*}{\rightarrow} u\right\} .
$$

LEMMA 2.3 The functional $\tilde{F}_{\varepsilon}$ is continuous on $L^{\infty}(\Omega,[0,1])$ for the weak-* topology of $L^{\infty}(\Omega)$.

Proof. We first note that $L^{\infty}(\Omega,[0,1])$, endowed with the weak-* topology of $L^{\infty}(\Omega)$, is metrizable. Thus continuity is equivalent to sequential continuity. Assume that $u_{n}, u \in$ $L^{\infty}(\Omega,[0,1])$ satisfy $u_{n} \rightarrow u$ weakly-* in $L^{\infty}(\Omega)$. Set $v_{n}=\left(-\varepsilon^{2} \Delta+I\right)^{-1} u_{n}$ and $v=$ $\left(-\varepsilon^{2} \Delta+I\right)^{-1} u$, so that by Proposition 2.1

$$
\tilde{F}_{\varepsilon}\left(u_{n}\right)=\frac{1}{2 \varepsilon}\left\langle 1-v_{n}, u_{n}\right\rangle, \quad \tilde{F}_{\varepsilon}(u)=\frac{1}{2 \varepsilon}\langle 1-v, u\rangle .
$$

For all test function $\varphi \in L^{2}(\Omega)$ we have, as the operator $\left(-\varepsilon^{2} \Delta+I\right)^{-1}$ is self-adjoint in $L^{2}(\Omega)$,

$$
\left\langle v_{n}, \varphi\right\rangle=\left\langle u_{n},\left(-\varepsilon^{2} \Delta+I\right)^{-1} \varphi\right\rangle \rightarrow\left\langle u,\left(-\varepsilon^{2} \Delta+I\right)^{-1} \varphi\right\rangle=\langle v, \varphi\rangle,
$$

hence $v_{n} \rightarrow v$ weakly-* in $L^{2}(\Omega)$. By standard elliptic operator theory, $\left\|v_{n}\right\|_{H^{1}(\Omega)}$ is uniformly bounded. By the Rellich theorem, one can extract a non-relabeled subsequence such that $v_{n} \rightarrow w$ strongly in $L^{2}(\Omega)$, for some $w \in L^{2}(\Omega)$. By uniqueness of the weak limit, we have $w=v$ and strong convergence of the whole sequence $\left(v_{n}\right)$. Finally, as product of strongly and weakly convergent sequences, we get $\left\langle v_{n}, u_{n}\right\rangle \rightarrow\langle v, u\rangle$, and subsequently $\tilde{F}_{\varepsilon}\left(u_{n}\right) \rightarrow \tilde{F}_{\varepsilon}(u)$.

Proposition 2.4 The function $\tilde{F}_{\varepsilon}: L^{\infty}(\Omega,[0,1]) \rightarrow \mathbb{R}$ is the relaxation of the functional $\bar{F}_{\varepsilon}$ defined by (2.11) with respect to the weak-* topology of $L^{\infty}(\Omega)$.

Proof. According to Proposition 11.1.1 of [13], the problem amounts to establishing the two following assertions:

$$
\begin{gathered}
\forall\left(u_{n}\right) \in L^{\infty}(\Omega,[0,1]), u_{n} \stackrel{*}{\rightarrow} u \Rightarrow \tilde{F}_{\varepsilon}(u) \leqslant \liminf _{n \rightarrow \infty} \bar{F}_{\varepsilon}\left(u_{n}\right), \\
\forall u \in L^{\infty}(\Omega,[0,1]) \exists\left(u_{n}\right) \in L^{\infty}(\Omega,[0,1]) \text { s.t. } u_{n} \stackrel{*}{\rightarrow} u, \tilde{F}_{\varepsilon}(u)=\lim _{n \rightarrow \infty} \bar{F}_{\varepsilon}\left(u_{n}\right) .
\end{gathered}
$$

Using that $\bar{F}_{\varepsilon}(u) \geqslant \tilde{F}_{\varepsilon}(u)$ for all $u \in L^{\infty}(\Omega,[0,1])$, the first assertion is a straightforward consequence of Lemma 2.3. Let now $u \in L^{\infty}(\Omega,[0,1])$ be arbitrary. A standard construction (see e.g. [25] proposition 7.2.14) enables to define a sequence $\left(u_{n}\right) \in L^{\infty}(\Omega,\{0,1\})$ such that $u_{n} \stackrel{*}{\rightarrow} u$. By Lemma 2.3 there holds

$$
\tilde{F}_{\varepsilon}(u)=\lim _{n \rightarrow \infty} \tilde{F}_{\varepsilon}\left(u_{n}\right)=\lim _{n \rightarrow \infty} \bar{F}_{\varepsilon}\left(u_{n}\right)
$$


We end this section by the explicit study of a typical one-dimensional example.

PROPOSITION 2.5 Let $a<0<b, \Omega=] a, b\left[\right.$ and $u=\chi_{] 0, b[}$. We have

$$
\begin{gathered}
\lim _{\varepsilon \rightarrow 0} F_{\varepsilon}(u)=\frac{1}{4}, \\
\frac{d}{d \varepsilon} F_{\varepsilon}(u) \leqslant 0, \quad \forall \varepsilon>0 .
\end{gathered}
$$

Proof. In order to solve (2.4) we make the splitting $v_{\varepsilon}=v_{\varepsilon, 1}+v_{\varepsilon, 2}$ with $v_{\varepsilon, 1}$ and $v_{\varepsilon, 2}$ respectively solutions of

$$
\begin{gathered}
-\varepsilon^{2} v_{\varepsilon, 1}^{\prime \prime}+v_{\varepsilon, 1}=\chi_{\mathbb{R}_{+}} \text {on } \mathbb{R}, \\
\left\{\begin{array}{c}
-\varepsilon^{2} v_{\varepsilon, 2}^{\prime \prime}+v_{\varepsilon, 2}=0 \text { on }[a, b], \\
v_{\varepsilon, 2}^{\prime}(a)=-v_{\varepsilon, 1}^{\prime}(a), v_{\varepsilon, 2}^{\prime}(b)=-v_{\varepsilon, 1}^{\prime}(b) .
\end{array}\right.
\end{gathered}
$$

We find the explicit solutions

$$
\begin{gathered}
v_{\varepsilon, 1}(x)= \begin{cases}\frac{1}{2} e^{x / \varepsilon} & \text { if } x \leqslant 0, \\
1-\frac{1}{2} e^{-x / \varepsilon} & \text { if } x \geqslant 0,\end{cases} \\
v_{\varepsilon, 2}(x)=-\frac{1}{2} \frac{e^{-2 a / \varepsilon}-1}{e^{2(b-a) / \varepsilon}-1} e^{x / \varepsilon}+\frac{1}{2} \frac{e^{2 b / \varepsilon}-1}{e^{2(b-a) / \varepsilon}-1} e^{-x / \varepsilon}, \quad \forall x \in \mathbb{R} .
\end{gathered}
$$

After some algebra we arrive at

$$
F_{\varepsilon}(u)=\frac{1}{4} \frac{\left(e^{-2 a / \varepsilon}-1\right)\left(e^{2 b / \varepsilon}-1\right)}{e^{2(b-a) / \varepsilon}-1} .
$$

Setting $t=2 / \varepsilon$, we obtain

$$
F_{\varepsilon}(u)=\frac{1}{4} \frac{\left(e^{-t a}-1\right)\left(e^{t b}-1\right)}{e^{t(b-a)}-1}=\frac{1}{4} \frac{\left(1-e^{t a}\right)\left(1-e^{-t b}\right)}{1-e^{-t(b-a)}} .
$$

Clearly, $F_{\varepsilon}(u) \rightarrow 1 / 4$ as $t \rightarrow+\infty$. Set now $\left.h=b-a>0, r=-a /(b-a) \in\right] 0,1[$, so that $a=-r h, b=(1-r) h$, and

$$
F_{\varepsilon}(u)=\frac{1}{4} \frac{\left(1-e^{-t r h}\right)\left(1-e^{-t(1-r) h}\right)}{1-e^{-t h}} .
$$

The change of variable $s=e^{-t h}$ leads to

$$
F_{\varepsilon}(u)=\frac{1}{4} \frac{\left(1-s^{r}\right)\left(1-s^{1-r}\right)}{1-s} .
$$

Now differentiating with respect to $s$ yields

$$
\frac{d}{d s} F_{\varepsilon}(u)=\frac{1}{4(1-s)^{2}}\left[2-r\left(s^{r-1}+s^{1-r}\right)-(1-r)\left(s^{-r}+s^{r}\right)\right] .
$$

Set

$$
f(s, r)=\frac{1}{2}\left[r\left(s^{r-1}+s^{1-r}\right)+(1-r)\left(s^{-r}+s^{r}\right)\right] .
$$


We have

$$
f\left(e^{\tau}, r\right)=r \cosh ((1-r) \tau)+(1-r) \cosh (r \tau)=: g_{r}(\tau)
$$

For fixed $r \in] 0,1$, the function $g_{r}$ is clearly even and nondecreasing on $\mathbb{R}_{+}$. Hence $g_{r}(\tau) \geqslant$ $g_{r}(0)=1$ for all $\tau \in \mathbb{R}$. This implies that $f(s, r) \geqslant 1$ for all $\left.(s, r) \in \mathbb{R}_{+}^{*} \times\right] 0,1[$, therefore

$$
\left.\frac{d}{d s} F_{\varepsilon}(u) \leqslant 0, \quad \forall(s, r) \in \mathbb{R}_{+}^{*} \times\right] 0,1[.
$$

Recalling that $s=e^{-2 h / \varepsilon}$, we derive

$$
\frac{d}{d \varepsilon} F_{\varepsilon}(u) \leqslant 0, \quad \forall \varepsilon>0 .
$$

\section{3. $\Gamma$-convergence of the approximating functionals}

This section addresses the $\Gamma$-convergence of the sequence of functionals $\left(\tilde{F}_{\varepsilon}\right)$ when $\varepsilon \rightarrow 0$. Note that, when a sequence is indexed by the letter $\varepsilon$, we actually mean any sequence of indices $\left(\varepsilon_{k}\right)$ of positive numbers going to zero.

\subsection{Definition and basic properties of the $\Gamma$-convergence}

The notion of $\Gamma$-convergence (see, e.g., $[13,18,20]$ ) is a powerful tool of calculus of variations in function spaces. Given a metrizable space $(X, d)$ (in our case $X=L^{\infty}(\Omega,[0,1])$ endowed with the distance induced by the $L^{1}$ norm) one would like the maps

$$
F \mapsto \inf _{X} F \quad \text { and } \quad F \mapsto \underset{X}{\operatorname{argmin} F}
$$

to be sequentially continuous on the space of extended real-valued functions $F: X \rightarrow \mathbb{R} \cup\{+\infty\}$.

Definition 3.1 Let $\left(\tilde{F}_{\varepsilon}\right)$ be a sequence of functions $\tilde{F}_{\varepsilon}: X \rightarrow \mathbb{R} \cup\{+\infty\}$ and $\tilde{F}: X \rightarrow$ $\mathbb{R} \cup\{+\infty\}$. We say that $\tilde{F}_{\varepsilon} \Gamma$-converges to $\tilde{F}$ if and only if, for all $u \in X$, the two following conditions hold:

(1) for all sequences $\left(u_{\varepsilon}\right) \in X$ such that $d\left(u_{\varepsilon}, u\right) \rightarrow 0$ it holds $\tilde{F}(u) \leqslant \liminf _{\varepsilon \rightarrow 0} \tilde{F}_{\varepsilon}\left(u_{\varepsilon}\right)$,

(2) there exists a sequence $\left(\bar{u}_{\varepsilon}\right) \in X$ such that $d\left(\bar{u}_{\varepsilon}, u\right) \rightarrow 0$ and $\tilde{F}(u) \geqslant \underset{\varepsilon \rightarrow 0}{\limsup } \tilde{F}_{\varepsilon}\left(\bar{u}_{\varepsilon}\right)$.

The key theorem we shall use in this paper is the following ( [13] Theorem 12.1.1).

TheOREM 3.2 Let $\tilde{F}_{\varepsilon}: X \rightarrow \mathbb{R} \cup\{+\infty\} \Gamma$-converge to $\tilde{F}: X \rightarrow \mathbb{R} \cup\{+\infty\}$.

(1) If $\left(u_{\varepsilon}\right)$ is a sequence of approximating minimizers for $\tilde{F}_{\varepsilon}$, i.e.

$$
\tilde{F}_{\varepsilon}\left(u_{\varepsilon}\right) \leqslant \inf _{u \in X} \tilde{F}_{\varepsilon}(u)+\lambda_{\varepsilon}
$$

with $\lambda_{\varepsilon} \rightarrow 0$, then $\inf _{u \in X} \tilde{F}_{\varepsilon}(u) \rightarrow \inf _{u \in X} \tilde{F}(u)$ and every cluster point of $\left(u_{\varepsilon}\right)$ is a minimizer of $\tilde{F}$.

(2) If $\tilde{J}: X \rightarrow \mathbb{R}$ is continuous, then $\tilde{J}+\tilde{F}_{\varepsilon} \Gamma$-converges to $\tilde{J}+\tilde{F}$.

Let us emphasize that the consideration of approximate minimizers is of major importance as soon as numerical approximations are made. 


\subsection{Preliminary results}

It turns out that the $\Gamma$-convergence can be straightforwardly deduced from the pointwise convergence if the sequence of functionals under consideration is nondecreasing and lower semicontinuous (see, e.g., [20] Proposition 5.4). Proposition 2.5 as well as several numerical tests based on the expression (2.7) of the derivative lead us to conjecture that $\tilde{F}_{\varepsilon}$ is indeed nondecreasing when $\varepsilon$ decreases. In addition, the pointwise convergence can be established, at least under some regularity assumptions, by harmonic analysis techniques, similarly to [10]. However, proving in full generality that (2.7) is nonpositive does not seem easy. We will proceed more directly.

We define the potential function $W: \mathbb{R} \rightarrow \mathbb{R}_{+}$by

$$
W(s)= \begin{cases}s(1-s) & \text { if } 0 \leqslant s \leqslant 1 \\ -s & \text { if } s \leqslant 0 \\ s-1 & \text { if } s \geqslant 1\end{cases}
$$

We set for all $u, v \in L^{1}(\Omega) \times L^{1}(\Omega)$

$\tilde{G}_{\varepsilon}(u, v)=\left\{\begin{array}{l}\frac{\varepsilon}{2}\|\nabla v\|_{L^{2}(\Omega)}^{2}+\frac{1}{2 \varepsilon}\|v-u\|_{L^{2}(\Omega)}^{2}+\frac{1}{2 \varepsilon} \int_{\Omega} W(u) d x \text { if }(u, v) \in L^{2}(\Omega) \times H^{1}(\Omega), \\ +\infty \\ \text { otherwise }\end{array}\right.$

Note that, if $(u, v) \in L^{\infty}(\Omega,[0,1]) \times H^{1}(\Omega)$, then

$$
\begin{aligned}
\tilde{G}_{\varepsilon}(u, v) & =\frac{\varepsilon}{2}\|\nabla v\|_{L^{2}(\Omega)}^{2}+\frac{1}{2 \varepsilon}\|v-u\|_{L^{2}(\Omega)}^{2}+\frac{1}{2 \varepsilon}\langle u, 1-u\rangle \\
& =\frac{\varepsilon}{2}\|\nabla v\|_{L^{2}(\Omega)}^{2}+\frac{1}{2 \varepsilon}\left(\|v\|_{L^{2}(\Omega)}^{2}+\langle u, 1-2 v\rangle\right) .
\end{aligned}
$$

Therefore we have for all $u \in L^{\infty}(\Omega,[0,1])$

$$
\tilde{F}_{\varepsilon}(u)=\inf _{v \in H^{1}(\Omega)} \tilde{G}_{\varepsilon}(u, v) .
$$

The following theorem, taken from [32] (see also [1]), will play a central role in our proof. We recall (see, e.g., [13]) that the total variation of $u \in L^{1}(\Omega)$ is defined as

$$
|D u|(\Omega)=\sup \left\{\langle u, \operatorname{div} \xi\rangle: \xi \in \mathrm{C}_{c}^{\infty}(\Omega)^{N},|\xi(x)| \leqslant 1 \forall x \in \Omega\right\},
$$

and $u$ is said of bounded variation, denoted $u \in B V(\Omega)$, when $|D u|(\Omega)<\infty$. When $u$ belongs to $B V(\Omega)$, its distributional derivative $D u$ is a Borel measure of total mass $|D u|(\Omega)$. If $u$ is the characteristic function of some subset $A$ of $\Omega$ with finite perimeter (i.e. $u$ is of bounded variation), then $|D u|(\Omega)$ corresponds to the relative perimeter of $A$ in $\Omega$, namely, the $N-1$ dimensional Hausdorff measure of $\partial A \cap \Omega$ (the boundary of $A$ is here meant in the geometric measure theory sense).

THEOREM 3.3 When $\varepsilon \rightarrow 0$, the functionals $\tilde{G}_{\varepsilon} \Gamma$-converge in $L^{1}(\Omega) \times L^{1}(\Omega)$ to the functional

$$
\tilde{G}(u, v)= \begin{cases}\kappa|D u|(\Omega) & \text { if } u=v \in B V(\Omega,\{0,1\}) \\ +\infty & \text { otherwise }\end{cases}
$$


The constant $\kappa$ is given by the two alternative expressions

$$
\begin{gathered}
\kappa=\frac{1}{2} \inf \left\{\int_{\mathbb{R}} W(\varphi) d x+\frac{1}{4} \int_{\mathbb{R}^{2}} e^{-|x-y|}(\varphi(x)-\varphi(y))^{2} d x d y, \varphi \in Y\right\}, \\
\kappa=\frac{1}{2} \inf \left\{\int_{\mathbb{R}} W(\varphi) d x+\int_{\mathbb{R}}\left[\left(\psi^{\prime}\right)^{2}+(\psi-\varphi)^{2}\right] d x, \varphi \in Y, \psi \in Y \cap H_{l o c}^{1}(\mathbb{R})\right\},
\end{gathered}
$$

with $Y=\left\{\varphi \in L^{\infty}(\mathbb{R},[0,1]), \varphi=\chi\right] 0,+\infty[$ on $\mathbb{R} \backslash]-R, R[$ for some $R>0\}$.

Before stating our $\Gamma$-convergence result for $\tilde{F}_{\varepsilon}$ (Theorem 3.7), we shall prove three technical lemmas useful for the proof.

LEMMA 3.4 Let $\Phi_{\varepsilon}$ be the fundamental solution of the operator $-\varepsilon^{2} \Delta+I$ on $\mathbb{R}^{N}$. For all $u \in$ $L^{1}\left(\mathbb{R}^{N},[0,1]\right)$ we have

$$
\lim _{\varepsilon \rightarrow 0}\left\|\Phi_{\varepsilon} * u-u\right\|_{L^{1}\left(\mathbb{R}^{N}\right)}=0 .
$$

Proof. Let $\lambda>0$ be arbitrary. A classical density result gives the existence of $v \in \mathbb{C}\left(\mathbb{R}^{N},[0,1]\right)$ with compact support such that $\|u-v\|_{L^{1}\left(\mathbb{R}^{N}\right)} \leqslant \lambda$. We have

$$
\left\|\Phi_{\varepsilon} * u-u\right\|_{L^{1}\left(\mathbb{R}^{N}\right)} \leqslant\left\|\Phi_{\varepsilon} * v-v\right\|_{L^{1}\left(\mathbb{R}^{N}\right)}+\left\|\Phi_{\varepsilon} *(u-v)\right\|_{L^{1}\left(\mathbb{R}^{N}\right)}+\|u-v\|_{L^{1}\left(\mathbb{R}^{N}\right)} .
$$

Using that $\Phi_{\varepsilon} \geqslant 0$ (from the maximum principle) and $\int_{\mathbb{R}^{N}} \Phi_{\varepsilon}=1$ (from $-\varepsilon^{2} \Delta \Phi_{\varepsilon}+\Phi_{\varepsilon}=\delta$ ), we obtain

$$
\left\|\Phi_{\varepsilon} * u-u\right\|_{L^{1}\left(\mathbb{R}^{N}\right)} \leqslant\left\|\Phi_{\varepsilon} * v-v\right\|_{L^{1}\left(\mathbb{R}^{N}\right)}+2\|u-v\|_{L^{1}\left(\mathbb{R}^{N}\right)} .
$$

Now let $\mu>0$. By uniform continuity of $v$ (Heine's theorem) there exists $\eta>0$ such that

$$
|x-y| \leqslant \eta \Rightarrow|v(x)-v(y)| \leqslant \mu .
$$

We have for any $x \in \mathbb{R}^{N}$

$$
\begin{aligned}
\left|\left(\Phi_{\varepsilon} * v-v\right)(x)\right|= & \left|\int_{\mathbb{R}^{N}} \Phi_{\varepsilon}(x-y)(v(y)-v(x)) d y\right| \\
\leqslant & \int_{\{|y-x| \leqslant \eta\}} \Phi_{\varepsilon}(x-y)|v(y)-v(x)| d y \\
& \quad+\int_{\{|y-x|>\eta\}} \Phi_{\varepsilon}(x-y)|v(y)-v(x)| d y \\
\leqslant & +\int_{\{|y-x|>\eta\}} \Phi_{\varepsilon}(x-y) d y .
\end{aligned}
$$

By change of variable we have $\Phi_{\varepsilon}(z)=\left(1 / \varepsilon^{N}\right) \Phi_{1}(z / \varepsilon)$, whereby $\int_{\{|y-x|>\eta\}} \Phi_{\varepsilon}(x-y) d y=$ $\int_{\{|z|>\eta / \varepsilon\}} \Phi_{1}(z) d z$. Therefore we get for $\varepsilon$ small enough $\int_{\{|y-x|>\eta\}} \Phi_{\varepsilon}(x-y) d y \leqslant \mu$. This shows that $\left|\left(\Phi_{\varepsilon} * v-v\right)(x)\right| \rightarrow 0$ uniformly on $\mathbb{R}^{N}$. This entails $\left\|\Phi_{\varepsilon} * v-v\right\|_{L^{1}\left(\mathbb{R}^{N}\right)} \rightarrow 0$. Consequently, we have for $\varepsilon$ small enough $\left\|\Phi_{\varepsilon} * v-v\right\|_{L^{1}\left(\mathbb{R}^{N}\right)} \leqslant \lambda$. Going back to (3.3) we arrive at

$$
\left\|\Phi_{\varepsilon} * u-u\right\|_{L^{1}\left(\mathbb{R}^{N}\right)} \leqslant 3 \lambda .
$$

As $\lambda$ is arbitrary this proves the desired convergence. 
We define the projection (or truncation) operator $P_{[0,1]}: \mathbb{R} \rightarrow[0,1]$ by

$$
P_{[0,1]}(s)=\max (0, \min (1, s)) .
$$

Lemma 3.5 Let $(u, v) \in L^{2}(\Omega) \times H^{1}(\Omega)$ and set $\tilde{u}=P_{[0,1]}(u), \tilde{v}=P_{[0,1]}(v)$. Then

$$
\tilde{G}_{\varepsilon}(\tilde{u}, \tilde{v}) \leqslant \tilde{G}_{\varepsilon}(u, v) .
$$

Proof. We shall show that each term in the definition of $\tilde{G}_{\varepsilon}$ is decreased by truncation. Suppose that $(u, v) \in L^{2}(\Omega) \times H^{1}(\Omega)$. For the first term we have $\nabla \tilde{v}=\chi_{\{0<v<1\}} \nabla v$. Hence $\|\nabla \tilde{v}\|_{L^{2}(\Omega)}^{2} \leqslant$ $\|\nabla v\|_{L^{2}(\Omega)}^{2}$. For the second term we use that the projection $P_{[0,1]}$ is 1-Lipschitz, which yields

$$
|\tilde{v}(x)-\tilde{u}(x)| \leqslant|v(x)-u(x)|, \quad \forall x \in \Omega .
$$

This obviously implies that $\|\tilde{v}-\tilde{u}\|_{L^{2}(\Omega)}^{2} \leqslant\|v-u\|_{L^{2}(\Omega)}^{2}$. As to the last term we notice that, by construction of $W$, we have

$$
0 \leqslant W\left(P_{[0,1]}(s)\right) \leqslant W(s), \quad \forall s \in \mathbb{R} .
$$

The last lemma addresses the value of the constant $\kappa$. Observe that Proposition 2.5 already suggests that $\kappa=1 / 4$, since $\kappa$ is independent of the dimension. Nevertheless, as the $\Gamma$-limit and the pointwise limit do not necessarily coincide, a proof remains to be done.

Lemma 3.6 For the potential $W$ given by (3.1), the constant $\kappa$ of Theorem 3.3 is $\kappa=1 / 4$.

Proof. Starting from the second expression of $\kappa$ we arrive at:

$$
\kappa=\inf \left\{\mathcal{F}(\varphi, \psi):=\frac{1}{2} \int_{\mathbb{R}}\left[\left(\psi^{\prime}\right)^{2}+\psi^{2}-2 \varphi \psi+\varphi\right] d x, \varphi \in Y, \psi \in Y \cap H_{l o c}^{1}(\mathbb{R})\right\} .
$$

For a given pair $(\varphi, \psi) \in Y \times\left(Y \cap H_{\text {loc }}^{1}(\mathbb{R})\right)$, it is observed that keeping $\psi$ fixed and defining $\tilde{\varphi}$ by $\tilde{\varphi}(x)=0$ if $\psi(x) \leqslant 1 / 2$ and $\tilde{\varphi}(x)=1$ if $\psi(x)>1 / 2$ provides a better candidate for the infimum. Therefore

$$
\kappa=\inf \left\{\mathcal{F}(\varphi, \psi), \varphi \in Y \cap L^{\infty}(\mathbb{R},\{0,1\}), \psi \in Y \cap H_{\text {loc }}^{1}(\mathbb{R})\right\} .
$$

We now argue similarly to [32] to show that it is enough to consider nondecreasing functions $\varphi$ and $\psi$. Let $(\varphi, \psi) \in L^{\infty}(\mathbb{R},\{0,1\}) \times L^{\infty}(\mathbb{R},[0,1])$ and $R>0$ be such that $\varphi=\psi=\chi_{0,+\infty[}$ on $\mathbb{R} \backslash]-R, R\left[\right.$. We define the right rearrangement of a subset $A$ of $[-R, R]$ by $A^{\#}=[R-|A|, R]$. If $f \in L^{\infty}([-R, R],[0,1])$ we set

$$
f^{\sharp}(x)=\sup \left\{\lambda \in \mathbb{R}, x \in I_{\lambda}^{\sharp}\right\}, \quad x \in[-R, R],
$$

with $I_{\lambda}=\{x \in[-R, R], f(x) \geqslant \lambda\}$. It directly stems from this definition that the $\lambda$ upper level-set of $f^{\#}$ is $I_{\lambda}^{\#}$. Hence $f^{\#}$ is always nondecreasing with values in $[\min f$, $\max f]$. Indeed, if $-R \leqslant x \leqslant$ $y \leqslant R$, then for any given $\lambda$ it holds $x \in\{f \geqslant \lambda\}^{\sharp} \Rightarrow y \in\{f \geqslant \lambda\}^{\sharp}$ whereby $f^{\sharp}(x) \geqslant \lambda \Rightarrow$ $f^{\sharp}(y) \geqslant \lambda$, since $\left\{f^{\sharp} \geqslant \lambda\right\}=\{f \geqslant \lambda\}^{\#}=I_{\lambda}^{\#}$. We set

$$
\bar{\varphi}(x)= \begin{cases}0 & \text { if } x<-R, \\ \varphi^{\sharp}(x) & \text { if }-R \leqslant x \leqslant R, \\ 1 & \text { if } R<x,\end{cases}
$$


and define $\bar{\psi}$ likewise. Standard properties of this type of rearrangement $[25,26,34]$ ensure that $\mathcal{F}(\bar{\varphi}, \bar{\psi}) \leqslant \mathcal{F}(\varphi, \psi)$. In addition, $\bar{\varphi}$ remains with values in $\{0,1\}$. Hence, denoting by $Y^{+}$the set of nondecreasing functions of $Y$, we have

$$
\kappa=\inf \left\{\mathcal{F}(\varphi, \psi), \varphi \in Y^{+} \cap L^{\infty}(\mathbb{R},\{0,1\}), \psi \in Y^{+} \cap H_{l o c}^{1}(\mathbb{R})\right\} .
$$

We now choose an arbitrary $\varphi \in Y^{+} \cap L^{\infty}(\mathbb{R},\{0,1\})$. Due to the invariance by translation of the functional $\mathcal{F}$, we may assume without any loss of generality that $\varphi=\chi_{\mathbb{R}_{+}}$. The function $\psi$ which minimizes $\mathcal{F}(\varphi,$.$) is solution to (2.12) with \varepsilon=1$, and its expression is given by (2.13). A short calculation results in $\kappa=\mathfrak{F}(\varphi, \psi)=1 / 4$.

\subsection{Main result}

With Theorem 3.3 and the three above lemmas at hand we are now able to state and prove our $\Gamma$-convergence result.

THEOREM 3.7 When $\varepsilon \rightarrow 0$, the functionals $\tilde{F}_{\varepsilon} \Gamma$-converge in $L^{\infty}(\Omega,[0,1])$ endowed with the strong topology of $L^{1}(\Omega)$ to the functional

$$
\tilde{F}(u)= \begin{cases}\frac{1}{4}|D u|(\Omega) & \text { if } u \in B V(\Omega,\{0,1\}), \\ +\infty & \text { otherwise }\end{cases}
$$

Proof. (1) Let $\left(u_{\varepsilon}\right), u \in L^{\infty}(\Omega,[0,1])$ be such that $u_{\varepsilon} \rightarrow u$ in $L^{1}(\Omega)$. For each $\varepsilon>0$ there exists a (unique) function $v_{\varepsilon} \in H^{1}(\Omega)$ such that $\tilde{F}_{\varepsilon}\left(u_{\varepsilon}\right)=\tilde{G}_{\varepsilon}\left(u_{\varepsilon}, v_{\varepsilon}\right)$. This is the solution of

$$
\begin{cases}-\varepsilon^{2} \Delta v_{\varepsilon}+v_{\varepsilon}=u_{\varepsilon} & \text { in } \Omega, \\ \partial_{n} v_{\varepsilon}=0 & \text { on } \partial \Omega .\end{cases}
$$

Set $w_{\varepsilon}=\Phi_{\varepsilon} * u_{\varepsilon}$, where $\Phi_{\varepsilon}$ is as in Lemma 3.4 and $u_{\varepsilon}$ is extended by zero outside $\Omega$. By the Lax-Milgram theorem we have

$$
\frac{1}{2}\left(\varepsilon^{2}\left\|\nabla v_{\varepsilon}\right\|_{L^{2}(\Omega)}^{2}+\left\|v_{\varepsilon}\right\|_{L^{2}(\Omega)}^{2}\right)-\left\langle u_{\varepsilon}, v_{\varepsilon}\right\rangle \leqslant \frac{1}{2}\left(\varepsilon^{2}\left\|\nabla w_{\varepsilon}\right\|_{L^{2}(\Omega)}^{2}+\left\|w_{\varepsilon}\right\|_{L^{2}(\Omega)}^{2}\right)-\left\langle u_{\varepsilon}, w_{\varepsilon}\right\rangle .
$$

Adding to both sides $\frac{1}{2}\left\|u_{\varepsilon}\right\|_{L^{2}(\Omega)}^{2}$ results in

$$
\varepsilon^{2}\left\|\nabla v_{\varepsilon}\right\|_{L^{2}(\Omega)}^{2}+\left\|v_{\varepsilon}-u_{\varepsilon}\right\|_{L^{2}(\Omega)}^{2} \leqslant \varepsilon^{2}\left\|\nabla w_{\varepsilon}\right\|_{L^{2}(\Omega)}^{2}+\left\|w_{\varepsilon}-u_{\varepsilon}\right\|_{L^{2}(\Omega)}^{2} .
$$

Yet the right hand side is bounded from above by

$$
\begin{aligned}
\varepsilon^{2}\left\|\nabla w_{\varepsilon}\right\|_{L^{2}\left(\mathbb{R}^{N}\right)}^{2}+\left\|w_{\varepsilon}-u_{\varepsilon}\right\|_{L^{2}\left(\mathbb{R}^{N}\right)}^{2} & =\int_{\mathbb{R}^{N}}\left[\left(-\varepsilon^{2} \Delta w_{\varepsilon}+w_{\varepsilon}\right) w_{\varepsilon}-2 u_{\varepsilon} w_{\varepsilon}+u_{\varepsilon}^{2}\right] d x \\
& =\int_{\mathbb{R}^{N}}\left[u_{\varepsilon} w_{\varepsilon}-2 u_{\varepsilon} w_{\varepsilon}+u_{\varepsilon}^{2}\right] d x \\
& =\int_{\mathbb{R}^{N}}\left(u_{\varepsilon}-w_{\varepsilon}\right) u_{\varepsilon} d x .
\end{aligned}
$$


We obtain

$$
\begin{aligned}
\left\|v_{\varepsilon}-u_{\varepsilon}\right\|_{L^{2}(\Omega)}^{2} & \leqslant\left\|u_{\varepsilon}-w_{\varepsilon}\right\|_{L^{1}(\Omega)} \\
& \leqslant\left\|u-\Phi_{\varepsilon} * u\right\|_{L^{1}(\Omega)}+\left\|u-u_{\varepsilon}\right\|_{L^{1}(\Omega)}+\left\|\Phi_{\varepsilon} *\left(u_{\varepsilon}-u\right)\right\|_{L^{1}(\Omega)} .
\end{aligned}
$$

By virtue of Lemma 3.4 and Fubini's theorem the right hand side goes to zero, hence $\| v_{\varepsilon}-$ $u_{\varepsilon} \|_{L^{2}(\Omega)} \rightarrow 0$. Next we have

$$
\left\|v_{\varepsilon}-u\right\|_{L^{1}(\Omega)} \leqslant\left\|v_{\varepsilon}-u_{\varepsilon}\right\|_{L^{1}(\Omega)}+\left\|u_{\varepsilon}-u\right\|_{L^{1}(\Omega)} \leqslant|\Omega|^{1 / 2}\left\|v_{\varepsilon}-u_{\varepsilon}\right\|_{L^{2}(\Omega)}+\left\|u_{\varepsilon}-u\right\|_{L^{1}(\Omega)} .
$$

It follows that $\left\|v_{\varepsilon}-u\right\|_{L^{1}(\Omega)} \rightarrow 0$. We infer using Theorem 3.3:

$$
\begin{aligned}
\liminf _{\varepsilon \rightarrow 0} \tilde{F}_{\varepsilon}\left(u_{\varepsilon}\right) & =\liminf _{\varepsilon \rightarrow 0} \tilde{G}_{\varepsilon}\left(u_{\varepsilon}, v_{\varepsilon}\right) \\
& \geqslant \tilde{G}(u, u)=4 \kappa \tilde{F}(u) .
\end{aligned}
$$

(2) Suppose that $u \in L^{\infty}(\Omega,[0,1])$. By Theorem 3.3 there exists $\left(u_{\varepsilon}, v_{\varepsilon}\right) \in L^{2}(\Omega) \times H^{1}(\Omega)$ such that $u_{\varepsilon} \rightarrow u, v_{\varepsilon} \rightarrow u$ in $L^{1}(\Omega)$, and

$$
\limsup _{\varepsilon \rightarrow 0} \tilde{G}_{\varepsilon}\left(u_{\varepsilon}, v_{\varepsilon}\right) \leqslant \tilde{G}(u, u) .
$$

By truncation (see Lemma 3.5), one may assume that $u_{\varepsilon}, v_{\varepsilon} \in L^{\infty}(\Omega,[0,1])$. Yet $\tilde{F}_{\varepsilon}\left(u_{\varepsilon}\right) \leqslant$ $\tilde{G}_{\varepsilon}\left(u_{\varepsilon}, v_{\varepsilon}\right)$, which entails

$$
\limsup _{\varepsilon \rightarrow 0} \tilde{F}_{\varepsilon}\left(u_{\varepsilon}\right) \leqslant \tilde{G}(u, u)=4 \kappa \tilde{F}(u) .
$$

(3) The value $\kappa=1 / 4$ obtained in Lemma 3.6 completes the proof.

\section{Solution of topology optimization problems with perimeter penalization}

In this section we propose solution methods for the optimization of shape functionals involving a perimeter term. The functionals under consideration will be of the form $j_{\alpha}(A)=J_{\alpha}\left(\chi_{A}\right)$, with

$$
J_{\alpha}(u)=J(u)+\frac{\alpha}{4}|D u|(\Omega) .
$$

The so-called "cost" or "objective" function $J$ is the quantity which we seek to optimize. Typically, in shape optimization, $J$ represents the flexibility (or compliance) of a load-bearing structure. In image processing $J$ may represent a distance between an observed image and the image to reconstruct. Usually, $J$ is a shape functional and it is known that its minimization without perimeter control will involve some relaxation of $J$. More generally, we consider here an arbitrary extension $\tilde{J}$ of $J$ to $L^{\infty}(\Omega,[0,1])$. Following our approach, $J_{\alpha}$ will be approximated through a continuation procedure by a sequence of auxiliary functionals of the form

$$
\tilde{J}_{\alpha, \varepsilon}(u)=\tilde{J}(u)+\alpha \tilde{F}_{\varepsilon}(u) .
$$

The issue is then to study the convergence (up to a subsequence) of sequences of minimizers of $\tilde{J}_{\alpha, \varepsilon}(u)$. As is well-known, the $\Gamma$-convergence of the functionals is not sufficient for this, since it does not guarantee the compactness of the sequence. Typically, compactness stems from an additional equicoercivity property $[18,20]$. We follow this approach and establish the equicoercivity of the functionals $\tilde{J}_{\alpha, \varepsilon}$ in Theorem 4.7. Related compactness results for non-local functionals can be found in [1]. 


\subsection{Preliminary results}

LEMMA 4.1 Let $\left(u_{\varepsilon}\right)$ be a sequence of $L^{\infty}(\Omega,[0,1])$ such that $\left(\tilde{F}_{\varepsilon}\left(u_{\varepsilon}\right)\right)$ is bounded. For each $\varepsilon>0$ let $v_{\varepsilon} \in H^{1}(\Omega)$ be the solution of (3.6). Then $\left(v_{\varepsilon}\right)$ admits a subsequence which converges strongly in $L^{1}(\Omega)$.

Proof. We have by definition

$$
\tilde{F}_{\varepsilon}\left(u_{\varepsilon}\right)=\frac{\varepsilon}{2}\left\|\nabla v_{\varepsilon}\right\|_{L^{2}(\Omega)}^{2}+\frac{1}{2 \varepsilon}\left(\left\|v_{\varepsilon}\right\|_{L^{2}(\Omega)}^{2}+\left\langle u_{\varepsilon}, 1-2 v_{\varepsilon}\right\rangle\right),
$$

and, as $0 \leqslant u_{\varepsilon} \leqslant 1$,

$$
\left\langle u_{\varepsilon}, 1-2 v_{\varepsilon}\right\rangle \geqslant \int_{\Omega} \min \left(0,1-2 v_{\varepsilon}\right) d x
$$

Setting

$$
W(s)=s^{2}+\min (0,1-2 s)
$$

we obtain

$$
\tilde{F}_{\varepsilon}\left(u_{\varepsilon}\right) \geqslant \int_{\Omega}\left(\frac{\varepsilon}{2}\left|\nabla v_{\varepsilon}\right|^{2}+\frac{1}{2 \varepsilon} w\left(v_{\varepsilon}\right)\right) d x .
$$

Straightforward calculations show that the function $W$ is nonnegative, symmetric with respect to $1 / 2$, and vanishes only in 0 and 1 (see Figure 1). We now use a classical argument due to Modica [27], which consists in applying successively to the right hand side of (4.1) the elementary Young inequality and the chain rule. This entails

$$
\tilde{F}_{\varepsilon}\left(u_{\varepsilon}\right) \geqslant \int_{\Omega}\left|\nabla v_{\varepsilon}\right| \sqrt{W\left(v_{\varepsilon}\right)} d x=\int_{\Omega}\left|\nabla w_{\varepsilon}\right| d x,
$$

where $\psi$ is an arbitrary primitive of $\sqrt{W}$ and $w_{\varepsilon}=\psi \circ v_{\varepsilon}$. The weak maximum principle implies that $0 \leqslant v_{\varepsilon} \leqslant 1$, hence $\psi(0) \leqslant w_{\varepsilon} \leqslant \psi(1)$. It follows that $\left(w_{\varepsilon}\right)$ is bounded in $L^{1}(\Omega)$. By the compact embedding of $B V(\Omega)$ into $L^{1}(\Omega),\left(w_{\varepsilon}\right)$ admits a subsequence which converges strongly in $L^{1}(\Omega)$ to some function $w$. By construction, $\psi$ is an increasing homeomorphism of $\mathbb{R}$ into itself. Denoting by $\psi^{-1}$ the inverse function, we have $v_{\varepsilon}=\psi^{-1} \circ w_{\varepsilon}$. Up to a subsequence, we have $w_{\varepsilon} \rightarrow w$ almost everywhere, thus $v_{\varepsilon} \rightarrow \psi^{-1} \circ w=: v$ almost everywhere. The Lebesgue dominated convergence theorem yields that $v_{\varepsilon} \rightarrow v$ in $L^{1}(\Omega)$.

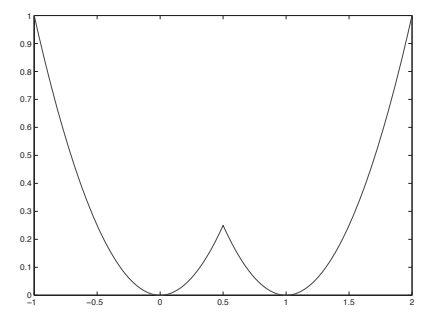

FIG. 1. Plot of the function $w$ 
LEMma 4.2 Let $\left(u_{\varepsilon}\right)$ be a sequence of $L^{\infty}(\Omega,[0,1])$ which converges weakly-* in $L^{\infty}(\Omega)$ to $u \in L^{\infty}(\Omega,[0,1])$. For each $\varepsilon>0$ let $v_{\varepsilon} \in H^{1}(\Omega)$ be the solution of (3.6). Then $v_{\varepsilon} \rightarrow u$ weakly in $L^{2}(\Omega)$.

Proof. The variational formulation for $v_{\varepsilon}$ reads

$$
\int_{\Omega}\left(\varepsilon^{2} \nabla v_{\varepsilon} \cdot \nabla \varphi+v_{\varepsilon} \varphi\right) d x=\int_{\Omega} u_{\varepsilon} \varphi d x \quad \forall \varphi \in H^{1}(\Omega) .
$$

Choosing $\varphi=v_{\varepsilon}$ and using the Cauchy-Schwarz inequality yields

$$
\begin{aligned}
\varepsilon^{2}\left\|\nabla v_{\varepsilon}\right\|_{L^{2}(\Omega)}^{2}+\left\|v_{\varepsilon}\right\|_{L^{2}(\Omega)}^{2} & \leqslant\left\|u_{\varepsilon}\right\|_{L^{2}(\Omega)}\left\|v_{\varepsilon}\right\|_{L^{2}(\Omega)} \\
& \leqslant\left\|u_{\varepsilon}\right\|_{L^{2}(\Omega)} \sqrt{\varepsilon^{2}\left\|\nabla v_{\varepsilon}\right\|_{L^{2}(\Omega)}^{2}+\left\|v_{\varepsilon}\right\|_{L^{2}(\Omega)}^{2}},
\end{aligned}
$$

which results in

$$
\varepsilon^{2}\left\|\nabla v_{\varepsilon}\right\|_{L^{2}(\Omega)}^{2}+\left\|v_{\varepsilon}\right\|_{L^{2}(\Omega)}^{2} \leqslant\left\|u_{\varepsilon}\right\|_{L^{2}(\Omega)}^{2} \leqslant|\Omega| .
$$

In particular we infer that

$$
\left\|v_{\varepsilon}\right\|_{L^{2}(\Omega)} \leqslant \sqrt{|\Omega|}, \quad\left\|\nabla v_{\varepsilon}\right\|_{L^{2}(\Omega)} \leqslant \frac{\sqrt{|\Omega|}}{\varepsilon} .
$$

Coming back to (4.2) we derive that, for every $\varphi \in H^{1}(\Omega)$,

$$
\int_{\Omega} v_{\varepsilon} \varphi d x=\int_{\Omega} u_{\varepsilon} \varphi d x-\varepsilon^{2} \int_{\Omega} \nabla v_{\varepsilon} \cdot \nabla \varphi d x
$$

Passing to the limit, we get with the help of (4.3)

$$
\int_{\Omega} v_{\varepsilon} \varphi d x \rightarrow \int_{\Omega} u \varphi d x
$$

Choose now an arbitrary test function $\psi \in L^{2}(\Omega)$, and fix $\rho>0$. By density of $H^{1}(\Omega)$ in $L^{2}(\Omega)$, there exists $\varphi \in H^{1}(\Omega)$ such that $\|\varphi-\psi\|_{L^{2}(\Omega)} \leqslant \rho$. From (4.4), there exists $\eta>0$ such that

$$
\left|\int_{\Omega}\left(v_{\varepsilon}-u\right) \varphi d x\right| \leqslant \rho, \quad \forall \varepsilon<\eta
$$

We obtain for any $\varepsilon<\eta$

$$
\begin{aligned}
\left|\int_{\Omega}\left(v_{\varepsilon}-u\right) \psi d x\right| & \leqslant\left|\int_{\Omega}\left(v_{\varepsilon}-u\right) \varphi d x\right|+\left|\int_{\Omega} v_{\varepsilon}(\psi-\varphi) d x\right|+\left|\int_{\Omega} u(\psi-\varphi) d x\right| \\
& \leqslant \rho(1+2 \sqrt{|\Omega|}) .
\end{aligned}
$$

Hence $v_{\varepsilon} \rightarrow u$ weakly in $L^{2}(\Omega)$.

LEMmA 4.3 Let $\left(u_{\varepsilon}\right) \in L^{\infty}(\Omega,[0,1])$ be a sequence such that $u_{\varepsilon} \rightarrow u$ weakly-* in $L^{\infty}(\Omega)$. If $u \in L^{\infty}(\Omega,\{0,1\})$, then $u_{\varepsilon} \rightarrow u$ strongly in $L^{1}(\Omega)$. 
Proof. We have by definition

$$
\int_{\Omega}\left(u_{\varepsilon}-u\right) \varphi d x \rightarrow 0 \quad \forall \varphi \in L^{1}(\Omega) .
$$

Since $u \in L^{\infty}(\Omega,\{0,1\})$ and $u_{\varepsilon} \in L^{\infty}(\Omega,[0,1])$, we have

$$
\int_{\Omega}\left|u_{\varepsilon}-u\right| d x=\int_{\{u=0\}} u_{\varepsilon} d x+\int_{\{u=1\}}\left(1-u_{\varepsilon}\right) d x
$$

From (4.5) with $\varphi=\chi_{\{u=0\}}$ we get

$$
\int_{\{u=0\}} u_{\varepsilon} d x \rightarrow 0
$$

Choosing now $\varphi=\chi_{\{u=1\}}$ results in

$$
\int_{\{u=1\}}\left(1-u_{\varepsilon}\right) d x \rightarrow 0
$$

which completes the proof.

The three above lemmas can be summarized in the following Proposition.

PROPOSITION 4.4 Let $\left(u_{\varepsilon}\right)$ be a sequence of $L^{\infty}(\Omega,[0,1])$ such that $\left(\tilde{F}_{\varepsilon}\left(u_{\varepsilon}\right)\right)$ is bounded. For each $\varepsilon>0$ let $v_{\varepsilon} \in H^{1}(\Omega)$ be the solution of (2.4) with right hand side $u_{\varepsilon}$. If $u_{\varepsilon} \rightarrow u$ weakly-* in $L^{\infty}(\Omega)$ then, for some subsequence, there holds:

(1) $v_{\varepsilon} \rightarrow u$ strongly in $L^{1}(\Omega)$,

(2) $u \in L^{\infty}(\Omega,\{0,1\})$,

(3) $u_{\varepsilon} \rightarrow u$ strongly in $L^{1}(\Omega)$.

Proof. By Lemma 4.2, we have $v_{\varepsilon} \rightarrow u$ weakly in $L^{2}(\Omega)$, thus also weakly in $L^{1}(\Omega)$ since $\Omega$ is bounded. By Lemma 4.1, we have for a subsequence $v_{\varepsilon} \rightarrow v \in L^{\infty}(\Omega,[0,1])$ strongly in $L^{1}(\Omega)$, and subsequently by uniqueness of the weak limit we have $v=u$.

Next, we have in view of (2.6)

$$
\tilde{F}_{\varepsilon}\left(u_{\varepsilon}\right)=\frac{1}{2 \varepsilon} \int_{\Omega}\left(1-v_{\varepsilon}\right) u_{\varepsilon} d x .
$$

Therefore, the boundedness of $\left(\tilde{F}_{\varepsilon}\left(u_{\varepsilon}\right)\right)$ entails

$$
\int_{\Omega}\left(1-v_{\varepsilon}\right) u_{\varepsilon} d x \rightarrow 0
$$

Yet, there holds

$$
\int_{\Omega}\left(1-v_{\varepsilon}\right) u_{\varepsilon} d x-\int_{\Omega}(1-u) u d x=\int_{\Omega}\left(u_{\varepsilon}-u\right)(1-u) d x-\int_{\Omega} u_{\varepsilon}\left(v_{\varepsilon}-u\right) d x
$$

Since, on one hand, $u_{\varepsilon} \rightarrow u$ weakly-* in $L^{\infty}(\Omega)$ and, on the other hand, $v_{\varepsilon} \rightarrow u$ strongly in $L^{1}(\Omega)$ and $u_{\varepsilon} \in L^{\infty}(\Omega,[0,1])$, both integrals at the right hand side of the above equality tend to zero. We arrive at

$$
\int_{\Omega}(1-u) u d x=0
$$


In addition, due to the closedness of $L^{\infty}(\Omega,[0,1])$ in the weak-* topology of $L^{\infty}(\Omega)$, we have $u \in L^{\infty}(\Omega,[0,1])$. We infer that $u(x) \in\{0,1\}$ for almost every $x \in \Omega$.

Finally, Lemma 4.3 implies that $u_{\varepsilon} \rightarrow u$ strongly in $L^{1}(\Omega)$.

\subsection{Existence and convergence of minimizers}

Consider a functional $J: L^{\infty}(\Omega,\{0,1\}) \rightarrow B$, where $B$ is a bounded interval of $\mathbb{R}$, and a parameter $\alpha>0$. We want to solve the minimization problem

$$
I:=\inf _{u \in B V(\Omega,\{0,1\})}\left\{J(u)+\frac{\alpha}{4}|D u|(\Omega)\right\} .
$$

Proposition 4.5 Assume that $J$ is lower semi-continuous on $L^{\infty}(\Omega,\{0,1\})$ for the strong topology of $L^{1}(\Omega)$. Then the infimum in (4.6) is attained.

Proof. Let $\left(u_{n}\right) \in B V(\Omega,\{0,1\})$ be a minimizing sequence. By boundedness of $\Omega$ and definition of the objective functional, $\left\|u_{n}\right\|_{L^{1}(\Omega)}+\left|D u_{n}\right|(\Omega)$ is uniformly bounded. Therefore, due to the compact embedding of $B V(\Omega)$ into $L^{1}(\Omega)$, one can extract a subsequence (not relabeled) such that $u_{n} \rightarrow u$ in $L^{1}(\Omega)$, for some $u \in L^{1}(\Omega)$. In addition, for a further subsequence, $u_{n} \rightarrow u$ almost everywhere in $\Omega$, thus $u \in L^{\infty}(\Omega,\{0,1\})$. Using the sequential lower semi-continuity of $J$ and $u \mapsto|D u|(\Omega)$, we obtain

$$
J(u)+\frac{\alpha}{4}|D u|(\Omega) \leqslant \liminf _{n \rightarrow \infty} J\left(u_{n}\right)+\frac{\alpha}{4}\left|D u_{n}\right|(\Omega)=I .
$$

It follows that $u$ is a minimizer.

Let $\tilde{J}: L^{\infty}(\Omega,[0,1]) \rightarrow B$ be an extension of $J$, i.e., a function such that $\tilde{J}(u)=J(u)$ for all $u \in L^{\infty}(\Omega,\{0,1\})$. By Theorem 3.7 we have

$$
I=\inf _{u \in L^{\infty}(\Omega,[0,1])}\{\tilde{J}(u)+\alpha \tilde{F}(u)\} .
$$

Given $\varepsilon>0$ we introduce the approximate problem:

$$
I_{\varepsilon}:=\inf _{u \in L^{\infty}(\Omega,[0,1])}\left\{\tilde{J}(u)+\alpha \tilde{F}_{\varepsilon}(u)\right\} .
$$

It turns out (cf. Theorem 4.8), that the approximate subproblem (4.8) needs to be solved only approximately. The existence of exact minimizers is nevertheless an information of interest regarding the design and analysis of a solution method.

Proposition 4.6 Assume that $\tilde{J}$ is lower semi-continuous for the weak-* topology of $L^{\infty}(\Omega)$. Then the infimum in (4.8) is attained.

Proof. By Lemma 2.3, the functional $u \in L^{\infty}(\Omega,[0,1]) \rightarrow \tilde{J}(u)+\alpha \tilde{F}_{\varepsilon}(u)$ is lower semicontinuous for the weak-* topology of $L^{\infty}(\Omega)$. In addition, the set $L^{\infty}(\Omega,[0,1])$ is compact for the same topology. The claim results from standard arguments.

Thanks to Proposition 4.4 the so-called equicoercivity property might be formulated as follows. 
THEOREM 4.7 Consider a sequence $\left(u_{\varepsilon}\right) \in L^{\infty}(\Omega,[0,1])$ such that

$$
\tilde{J}\left(u_{\varepsilon}\right)+\alpha \tilde{F}_{\varepsilon}\left(u_{\varepsilon}\right) \leqslant I_{\varepsilon}+\lambda_{\varepsilon}
$$

with $\left(\lambda_{\varepsilon}\right)$ bounded. There exists $u \in L^{\infty}(\Omega,\{0,1\})$ and a subsequence of indices such that $u_{\varepsilon} \rightarrow u$ strongly in $L^{1}(\Omega)$.

Proof. By the limsup inequality of the $\Gamma$-convergence, there exists a sequence $\left(z_{\varepsilon}\right) \in$ $L^{\infty}(\Omega,[0,1])$ such that $z_{\varepsilon} \rightarrow 0$ in $L^{1}(\Omega)$ and $\tilde{F}_{\varepsilon}\left(z_{\varepsilon}\right) \rightarrow \tilde{F}(0)=0$. For this particular sequence we have

$$
\tilde{J}\left(u_{\varepsilon}\right)+\alpha \tilde{F}_{\varepsilon}\left(u_{\varepsilon}\right) \leqslant \tilde{J}\left(z_{\varepsilon}\right)+\alpha \tilde{F}_{\varepsilon}\left(z_{\varepsilon}\right)+\lambda_{\varepsilon},
$$

which entails that $\left(\tilde{F}_{\varepsilon}\left(u_{\varepsilon}\right)\right)$ is bounded.

Now, since $L^{\infty}(\Omega,[0,1])$ is weakly-* compact in $L^{\infty}(\Omega)$, there exists $u \in L^{\infty}(\Omega,[0,1])$ and a non-relabeled subsequence such that $u_{\varepsilon} \rightarrow u$ weakly-* in $L^{\infty}(\Omega)$. Using Proposition 4.4, we infer that $u \in L^{\infty}(\Omega,\{0,1\})$ as well as $u_{\varepsilon} \rightarrow u$ strongly in $L^{1}(\Omega)$.

Combining Theorem 3.2, Theorem 3.7 and Theorem 4.7 leads to the following result.

THEOREM 4.8 Let $\left(u_{\varepsilon}\right)$ is a sequence of approximating minimizers for (4.8), i.e., for each $\varepsilon>0$ $u_{\varepsilon} \in L^{\infty}(\Omega,[0,1])$ satisfies

$$
\tilde{J}\left(u_{\varepsilon}\right)+\alpha \tilde{F}_{\varepsilon}\left(u_{\varepsilon}\right) \leqslant I_{\varepsilon}+\lambda_{\varepsilon}
$$

with $\lim _{\varepsilon \rightarrow 0} \lambda_{\varepsilon}=0$. Assume that $\tilde{J}$ is continuous on $L^{\infty}(\Omega,[0,1])$ for the strong topology of $L^{1}(\Omega)$. Then we have $\tilde{J}\left(u_{\varepsilon}\right)+\alpha \tilde{F}_{\varepsilon}\left(u_{\varepsilon}\right) \rightarrow I$. Moreover, $\left(u_{\varepsilon}\right)$ admits cluster points for the strong topology of $L^{1}(\Omega)$, and each of these cluster points is a minimizer of (4.6).

Theorem 4.8 shows in particular that, when (4.6) admits a unique minimizer $u$, then the whole sequence $\left(u_{\varepsilon}\right)$ converges in $L^{1}(\Omega)$ to $u$. We have now a solid background to address the algorithmic issue.

\subsection{Algorithms for topology optimization with perimeter penalization}

As already said, we propose to use a continuation method with respect to $\varepsilon$. Namely, we construct a sequence $\left(\varepsilon_{k}\right)$ going to zero and solve at each iteration $k$ the minimization problem (4.8) using the previous solution as initial guess.

Several methods may be used to solve (4.8). The specific features of the functional $\tilde{J}$ may guide the choice.

(1) The most direct approach consists in using methods dedicated to the solution of optimization problem with box constraints, for instance the projected gradient method.

(2) When $\tilde{J}$ is continuous for the weak-* topology of $L^{\infty}(\Omega)$ one can restrict the feasible set to $L^{\infty}(\Omega,\{0,1\})$ and use topology optimization methods to find an approximate minimizer.

(3) Another alternative is to come back to the definition of $\tilde{F}_{\varepsilon}$ by (2.3), and write

$$
I_{\varepsilon}=\inf _{u \in L^{\infty}(\Omega,[0,1])} \inf _{v \in H^{1}(\Omega)}\left\{\tilde{J}(u)+\alpha\left[\frac{\varepsilon}{2}\|\nabla v\|_{L^{2}(\Omega)}^{2}+\frac{1}{2 \varepsilon}\left(\|v\|_{L^{2}(\Omega)}^{2}+\langle u, 1-2 v\rangle\right)\right]\right\} .
$$

Then one can use an alternating minimization algorithm with respect to the pair of variables $(u, v)$. 
In the subsequent sections we present three examples of application. The first one illustrates the method (1) in the context of least square problems. The last two ones deal with self-adjoint problems for which, as we shall see, the method (3) is particularly relevant. We refer to [10] for some examples of application of the approach (2).

For the discretization of all the PDEs involved, in particular (2.4), we use piecewise linear finite elements on a structured triangular mesh. We use the same finite elements to represent the variable $u$, although a piecewise constant approximation would be possible. This choice is motivated by two reasons. First, the expressions (2.3) or (2.6) of $\tilde{F}_{\varepsilon}(u)$ involve the scalar product $\langle u, v\rangle$, thus it is rather natural to approximate $u$ and $v$ with the same finite elements. Second, as observed in [11], the use of $P 1$ elements for $u$ cures the checkerboard instabilities, which otherwise typically occur when interpolation or homogenization methods are employed to solve minimal compliance problems with piecewise linear displacements $[2,15]$.

For each example different values of the penalization parameter $\alpha$ are considered. Note that choosing $\alpha$ too small requires, in order to eventually obtain a binary solution (i.e., in $L^{\infty}(\Omega,\{0,1\})$ ), to drive $\varepsilon$ towards very small values, which in turn necessitates the use of a very fine mesh to solve (2.4) with an acceptable accuracy. This is why, to enable comparisons of solutions obtained with identical meshes and a wide range of values of $\alpha$, we always use relatively fine meshes.

\section{First application: Source identification for the Poisson equation}

\subsection{Problem formulation}

For all $u \in L^{2}(\Omega)$ we denote by $y_{u} \in H_{0}^{1}(\Omega)$ the solution of

$$
\left\{\begin{array}{lll}
-\Delta y_{u}=u & \text { in } & \Omega \\
y_{u}=0 & \text { on } & \partial \Omega
\end{array}\right.
$$

and we set

$$
\tilde{J}(u)=\frac{1}{2}\left\|y_{u}-y^{\dagger}\right\|_{L^{2}(\Omega)}^{2},
$$

where $y^{\dagger} \in L^{2}(\Omega)$ is a given function.

Proposition 5.1 The functional $\tilde{J}$ is continuous on $L^{\infty}(\Omega,[0,1])$ strongly in $L^{1}(\Omega)$ and also weakly-* in $L^{\infty}(\Omega)$.

Proof. First we remark that if $\left(u_{n}\right)$ is a sequence of $L^{\infty}(\Omega,[0,1])$ such that $u_{n} \rightarrow u$ strongly in $L^{1}(\Omega)$, then $u_{n} \rightarrow u$ almost everywhere (for a subsequence), which implies that $u_{n} \rightarrow u$ weakly-* in $L^{\infty}(\Omega)$ by dominated convergence.

Thus, let us assume that $u_{n} \rightarrow u$ weakly-* in $L^{\infty}(\Omega)$. As $\left(\left\|y_{u_{n}}\right\|_{H^{1}(\Omega)}\right)$ is bounded, we can extract a subsequence such that $y_{u_{n}} \rightarrow y \in H_{0}^{1}(\Omega)$ weakly in $H_{0}^{1}(\Omega)$ and strongly in $L^{2}(\Omega)$. Passing to the limit in the weak formulation of (5.1), we obtain that $y=y_{u}$. Moreover, by uniqueness of this cluster point the whole sequence $\left(y_{u_{n}}\right)$ converges to $y$ for the aforementioned topologies. This implies that $\tilde{J}\left(u_{n}\right) \rightarrow \tilde{J}(u)$.

In consequence of these continuity properties, Proposition 4.5, Proposition 4.6 and Theorem 4.8 can be applied. 


\subsection{Algorithm and examples}

In our simulations $y^{\dagger}$ is defined by

$$
y^{\dagger}=y^{\sharp}+n,
$$

where $y^{\sharp}$ solves

$$
\left\{\begin{array}{lll}
-\Delta y^{\sharp}=u^{\sharp} & \text { in } & \Omega, \\
y^{\#}=0 & \text { on } & \partial \Omega,
\end{array}\right.
$$

for some given $u^{\sharp} \in L^{2}(\Omega)$ and $n \in L^{2}(\Omega)$. More precisely, $n$ is of the form $\beta \bar{n}$, with $\beta \geqslant 0$ and $\bar{n}(x)$ a random Gaussian noise with zero mean and unit variance. The function $u^{\sharp}$ is chosen as the characteristic function of a subdomain $\Omega^{\sharp} \subset \subset \Omega$.

The domain $\Omega$ is the unit square $] 0,1[\times] 0,1[$. We initialize $\varepsilon$ to 1 and divide it by 2 until it becomes less that $10^{-6}$. The initial guess is $u \equiv 1$. In order to solve the approximate problems we use a projected gradient method with line search. Here the mesh contains 80401 nodes. The results of computations performed with different values of the coefficients $\alpha$ and $\beta$ are depicted on Figure 2. Each plot represents the variable $u$ obtained at convergence. The absence of intermediate (grey) values is to be noticed. Rather than $\beta$, we indicate the noise to signal ratio, viz.,

$$
R=\frac{\|n\|_{L^{2}(\Omega)}}{\left\|y^{\dagger}\right\|_{L^{2}(\Omega)}} .
$$

We observe, as expected, that the higher the noise level is, the larger the penalization parameter $\alpha$ must be chosen in order to achieve a proper reconstruction. Of course, large values of $\alpha$ produce smoothed reconstructed shapes.

\section{Second application: Conductivity optimization}

\subsection{Problem formulation}

We consider a two-phase conductor $\Omega$ with source term $f \in L^{2}(\Omega)$. For all $u \in L^{\infty}(\Omega,[0,1])$ we define the conductivity

$$
\gamma_{u}:=\gamma_{0}(1-u)+\gamma_{1} u
$$

where $\gamma_{1}>\gamma_{0}>0$ are given constants. The objective functional is the power dissipated by the conductor augmented by a volume term, i.e.,

$$
\tilde{J}(u)=\int_{\Omega} f y d x+\ell \int_{\Omega} u d x
$$

where $\ell$ is a fixed positive multiplier and $y$ solves

$$
\left\{\begin{array}{lll}
-\operatorname{div}\left(\gamma_{u} \nabla y\right)=f & \text { in } & \Omega \\
y=0 & \text { on } & \partial \Omega
\end{array}\right.
$$

Note that the Dirichlet boundary condition has been chosen merely for simplicity of the presentation. Alternatively, this functional can be expressed in terms of the complementary energy (see, e.g., [3])

$$
\tilde{J}(u)=\inf _{\tau \in \Sigma}\left\{\int_{\Omega} \gamma_{u}^{-1}|\tau|^{2} d x\right\}+\ell \int_{\Omega} u d x,
$$




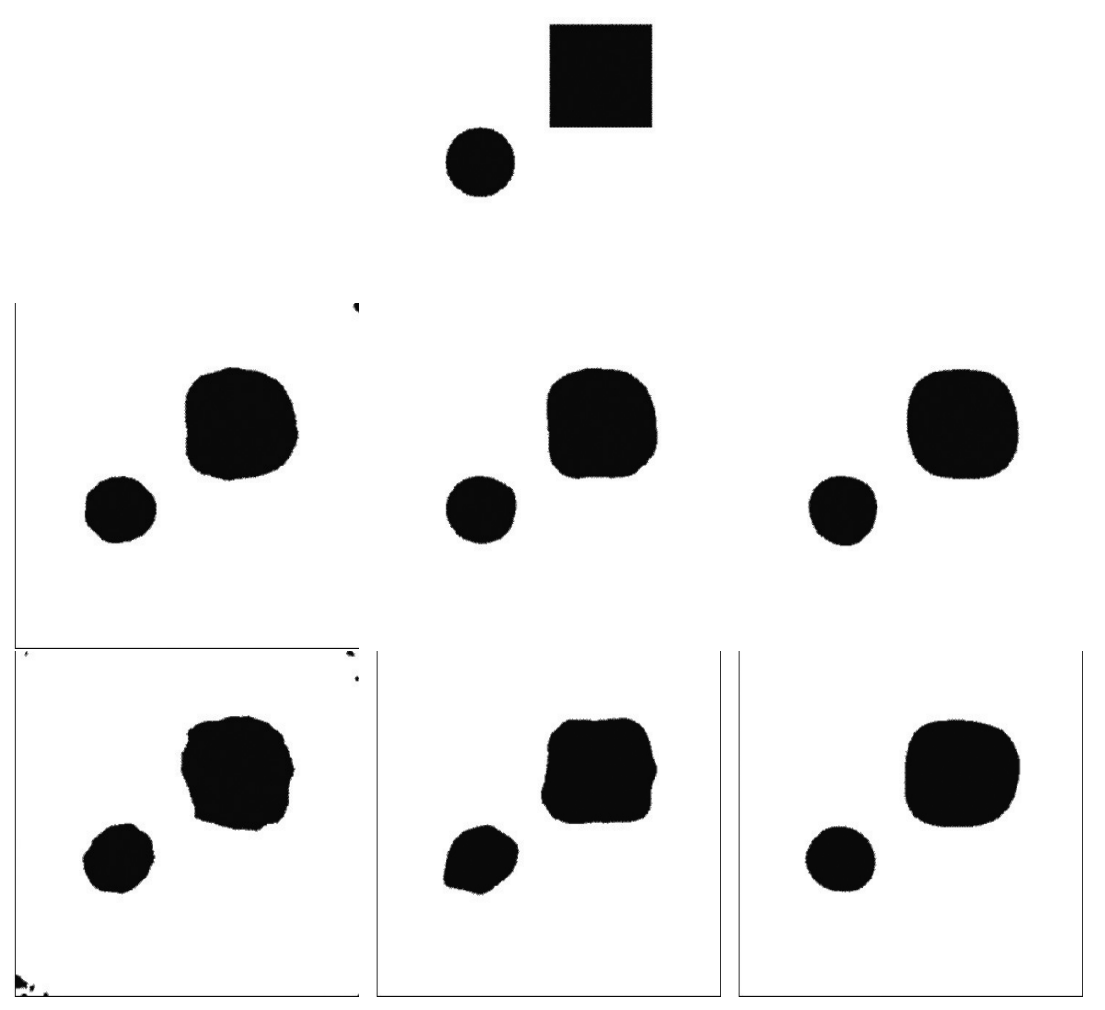

FIG. 2. Source identification. Top: True sources. Then reconstructed sources for $R=33 \%$ (first line) and $R=58 \%$ (second line) with $\alpha=10^{-8}$ (first column), $\alpha=10^{-7}$ (second column) and $\alpha=10^{-6}$ (third column).

with

$$
\Sigma=\left\{\tau \in L^{2}(\Omega)^{N},-\operatorname{div} \tau=f \text { in } \Omega\right\} .
$$

When it occurs that $u \in L^{\infty}(\Omega,\{0,1\})$ we set $J(u):=\tilde{J}(u)$. Given $\alpha>0$, we want to solve

$$
\inf _{u \in B V(\Omega,\{0,1\})}\left\{J(u)+\frac{\alpha}{4}|D u|(\Omega)\right\}
$$

which amounts to solving

$$
\inf _{u \in L^{\infty}(\Omega,[0,1])}\{\tilde{J}(u)+\alpha \tilde{F}(u)\}
$$

where $\tilde{F}(u)$ is defined by (3.5).

Proposition 6.1 The functional $\tilde{J}$ defined by (6.1) is continuous on $L^{\infty}(\Omega,[0,1])$ strongly in $L^{1}(\Omega)$.

Proof. Assume that $u_{n} \rightarrow u$ strongly in $L^{1}(\Omega)$, and denote by $y_{n}, y$ the corresponding states. Obviously, $\gamma_{u_{n}} \rightarrow \gamma_{u}$ strongly in $L^{1}(\Omega)$. Then $y_{n} \rightarrow y$ weakly in $H_{\tilde{\sigma}}^{1}(\Omega)$, see [13] Theorem 16.4.1 or [2] Lemma 1.2.22. It follows straightforwardly that $\tilde{J}\left(u_{n}\right) \rightarrow \tilde{J}(u)$. 
For $\varepsilon>0$ fixed we solve the approximate problem

$$
\inf _{u \in L^{\infty}(\Omega,[0,1])}\left\{\tilde{J}(u)+\alpha \tilde{F}_{\varepsilon}(u)\right\} .
$$

Using (2.3) and (6.3), this can be rewritten as

$$
\begin{aligned}
\inf _{(u, v, \tau) \in L^{\infty}(\Omega,[0,1]) \times H^{1}(\Omega) \times \Sigma}\left\{\int_{\Omega} \gamma_{u}^{-1}|\tau|^{2} d x+\ell \int_{\Omega} u d x+\right. \\
\left.\alpha\left[\frac{\varepsilon}{2}\|\nabla v\|_{L^{2}(\Omega)}^{2}+\frac{1}{2 \varepsilon}\left(\|v\|_{L^{2}(\Omega)}^{2}+\langle u, 1-2 v\rangle\right)\right]\right\} .
\end{aligned}
$$

In the proof of the following existence result we will use the notion of $G$-convergence (see e.g. [2]). We recall that a sequence of symmetric positive definite matrix fields $A_{n}$ is said to $G$ converge to $A$ if, for any right hand side $\varphi \in H^{-1}(\Omega)$, the sequence $\left(y_{n}\right)$ of solutions of

$$
\left\{\begin{array}{lll}
-\operatorname{div}\left(A_{n} \nabla y_{n}\right)=\varphi & \text { in } & \Omega \\
y_{n}=0 & \text { on } & \partial \Omega
\end{array}\right.
$$

converges weakly in $H_{0}^{1}(\Omega)$ to the solution $y$ of the so-called homogenized problem

$$
\left\{\begin{array}{lll}
-\operatorname{div}(A \nabla y)=\varphi & \text { in } & \Omega \\
y=0 & \text { on } & \partial \Omega
\end{array}\right.
$$

Proposition 6.2 The infima (6.5) and (6.6) are attained.

Proof. Since the infima (2.3) and (6.3) are both attained, it suffices to consider (6.5). Let therefore $\left(u_{n}\right)$ be a minimizing sequence for (6.5), whose corresponding solutions of (6.2) are denoted by $\left(y_{n}\right)$. We extract a subsequence, still denoted $\left(u_{n}\right)$, such that $u_{n} \rightarrow u \in L^{\infty}(\Omega,[0,1])$ weakly-* in $L^{\infty}(\Omega)$. By the so-called compactness property of the $G$-convergence (see, e.g., [2] Theorem 1.2.16), we can extract a further subsequence such that the matrix-valued conductivity $\gamma u_{n} I$, where $I$ is the identity matrix of order $N, G$-converges to some $A$, where, at each $x \in \Omega, A(x)$ is a symmetric positive definite $N \times N$ matrix. This means that $y_{n} \rightarrow y$ weakly in $H_{0}^{1}(\Omega)$, where $y$ solves

$$
\left\{\begin{array}{lll}
-\operatorname{div}(A \nabla y)=f & \text { in } & \Omega \\
y=0 & \text { on } & \partial \Omega .
\end{array}\right.
$$

By virtue of [2] Theorem 3.2.6, we have $A \nabla y=\gamma_{u} \nabla y$ at each point $x \in \Omega$. Therefore, by uniqueness, $y$ is the state associated to $u$. By $(6.1), \tilde{J}\left(u_{n}\right) \rightarrow \tilde{J}(u)$ while we know by Lemma 2.3 that $\tilde{F}_{\varepsilon}\left(u_{n}\right) \rightarrow \tilde{F}_{\varepsilon}(u)$. This completes the proof.

\subsection{Description of the algorithm}

In the spirit of [4], we use an alternating minimization algorithm, by performing successively a full minimization of (6.6) with respect to each of the variables $\tau, v, u$. The minimization with respect to $\tau$ is equivalent to solving (6.2) and setting $\tau=\gamma_{u} \nabla y$. The minimization with respect to $v$ is done by solving (2.4). Let us focus on the minimization with respect to $u$. We have to solve

$$
\inf _{u \in L \infty(\Omega,[0,1])}\left\{\int_{\Omega} \Phi_{\varepsilon, v, \tau}(u(x)) d x\right\}, \quad \text { with } \Phi_{\varepsilon, v, \tau}(u)=\gamma_{u}^{-1}|\tau|^{2}+\ell u+\frac{\alpha}{2 \varepsilon} u(1-2 v) .
$$


This means that, at every point $x \in \Omega$, we have to minimize the function $s \in[0,1] \mapsto \Phi_{\varepsilon, v, \tau}(s)$. From

$$
\Phi_{\varepsilon, v, \tau}(s)=\frac{|\tau|^{2}}{\gamma_{0}+\left(\gamma_{1}-\gamma_{0}\right) s}+\left[\ell+\frac{\alpha}{2 \varepsilon}(1-2 v)\right] s
$$

we readily find the minimizer

$$
u= \begin{cases}1 & \text { if } \ell+\frac{\alpha}{2 \varepsilon}(1-2 v) \leqslant 0, \\ P_{[0,1]}\left(\sqrt{\left.\frac{|\tau|^{2}}{\left(\gamma_{1}-\gamma_{0}\right)\left(\ell+\frac{\alpha}{2 \varepsilon}(1-2 v)\right)}-\frac{\gamma_{0}}{\gamma_{1}-\gamma_{0}}\right)}\right. & \text { if } \ell+\frac{\alpha}{2 \varepsilon}(1-2 v)>0,\end{cases}
$$

where we recall that $P_{[0,1]}$ is the projection operator defined by (3.4).

The regularization parameter $\varepsilon$ is initialized to 1 . It is divided by two each time a (local) minimizer of (6.6) has been found by the alternating algorithm, more precisely, when the relative variation of $\tilde{J}(u)+\alpha \tilde{F}_{\varepsilon}(u)$ between two iterations becomes less than some threshold $\Delta_{\max }$. The whole procedure is stopped when $\varepsilon$ becomes less that $h / 10$, with $h$ the mesh size.

\section{$6.3 \quad$ Numerical examples}

Our first example is a conductor with one inlet and two outlets, see Figure 3. The domain $\Omega$ is the square ]0, $1.5[\times] 0,1.5\left[\right.$. The conductivities of the two phases are $\gamma_{0}=10^{-3}$ and $\gamma_{1}=1$. The Lagrange multiplier is $\ell=2$. We use a mesh with 65161 nodes and the stopping criterion $\Delta_{\max }=10^{-3}$. The results of computations performed with different values of $\alpha$ are shown on Figure 4.

Our second example is a variant of the optimal heater problem presented in [3], with the boundary conditions slightly modified to avoid boundary effects caused by the relative perimeter (see Figure 5). The data are $\gamma_{0}=10^{-2}, \gamma_{1}=1$ and $\ell=10$. In order to capture fine details, we take a mesh with 115681 nodes and as stopping criterion $\Delta_{\max }=10^{-5}$. The results are depicted on Figure 6. A convergence history of the criterion $\tilde{J}(u)+\alpha \tilde{F}_{\varepsilon}(u)$ is given on Figure 7. The iterations where the criterion increases correspond to updates of $\varepsilon$. We observe that, when $\varepsilon$ becomes too small (with respect to the mesh size), the criterion increases much more during the updates of $\varepsilon$ than it decreases in between, while the design variables almost no longer evolve. This is a pure discretization effect, already mentioned, corresponding to the fact that the accuracy allowed by the mesh has been reached. There is no use to iterate further.

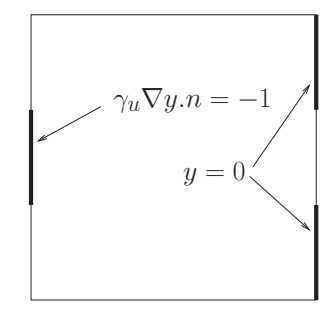

FIG. 3. Boundary conditions for the V-shaped conductor. An homogeneous Neumann condition is prescribed on the nonspecified boundaries. 

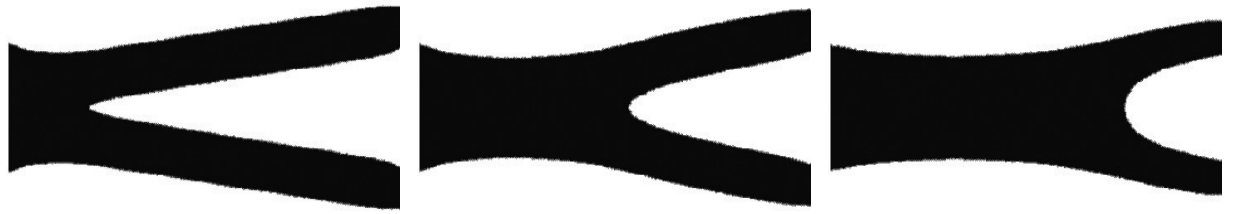

FIG. 4. Optimized V-shaped conductor for $\alpha=0.1,0.5,3$, respectively

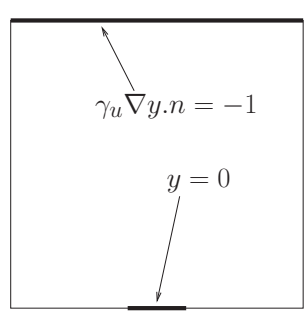

FIG. 5. Boundary conditions for the optimal heater problem
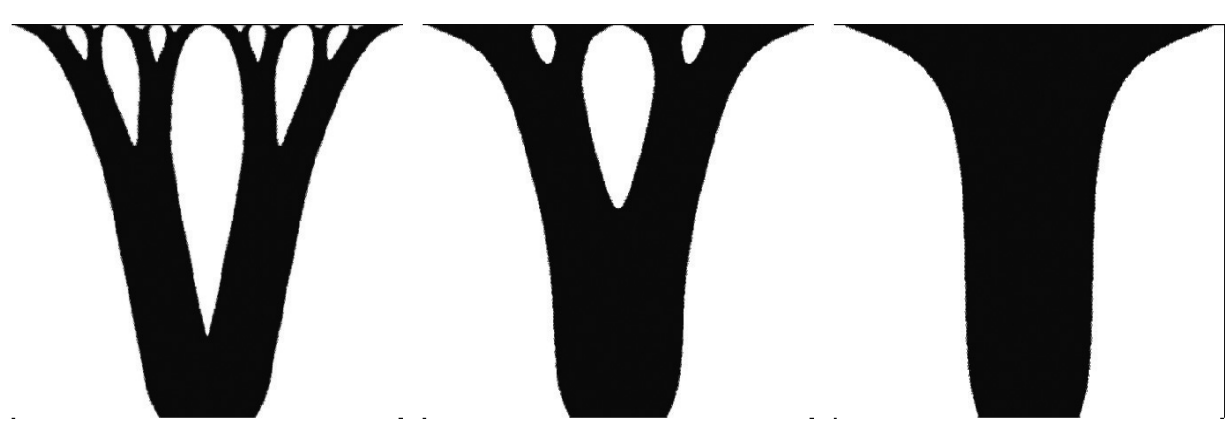

FIG. 6. Optimized heater for $\alpha=0.1,0.5,2$, respectively

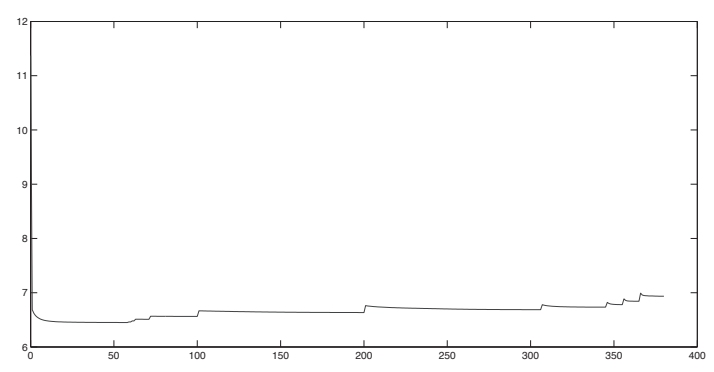

FIG. 7. Convergence history for the optimal heater problem $(\alpha=0.1)$, with the number of iterations on the $x$-axis and the values of the criterion on the y-axis. 


\section{Third application: Compliance minimization in linear elasticity}

\subsection{Problem formulation}

We assume now that $\Omega$ is occupied by a linear elastic material subject to a volume force $f \in$ $L^{2}(\Omega)^{N}$. We denote by $A(x)$ the Hooke tensor at point $x$. In the presentation we assume for simplicity, but without loss of generality, that the medium is clamped on $\partial \Omega$. The compliance can be defined either by

$$
C(A)=\int_{\Omega} f \cdot y d x
$$

where the displacement $y$ solves

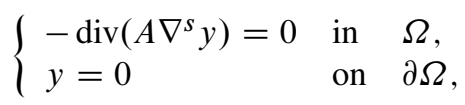

with $\nabla^{s}$ the symmetrized gradient, or with the help of the complementary energy [3],

$$
C(A)=\inf _{\sigma \in \Sigma}\left\{\int_{\Omega} A^{-1} \sigma: \sigma d x\right\}
$$

with

$$
\Sigma=\left\{\sigma \in L^{2}(\Omega)^{N \times N},-\operatorname{div} \sigma=f \text { in } \Omega\right\} .
$$

Given $\ell, \alpha>0$, we want to solve

$$
\inf _{u \in L^{\infty}(\Omega,\{0,1\})}\left\{J(u)+\frac{\alpha}{4}|D u|(\Omega)\right\},
$$

with

$$
J(u)=C(A(u))+\ell \int_{\Omega} u d x, \quad A(u)(x)=\left\{\begin{array}{lll}
A_{0} & \text { if } & u(x)=0 \\
A_{1} & \text { if } & u(x)=1
\end{array}\right.
$$

Here, $A_{0}, A_{1}$ are given Hooke tensors. Typically, $A_{1}$ corresponds to a physical material, while $A_{0}$ represents a weak phase of small Young modulus meant to mimick void. The problem can be reformulated as

$$
\inf _{u \in L^{\infty}(\Omega,[0,1])}\{\tilde{J}(u)+\alpha \tilde{F}(u)\}
$$

where

$$
\tilde{J}(u)=\inf _{A \in G_{u}} C(A)+\ell \int_{\Omega} u d x,
$$

the convention $A \in G_{u} \Longleftrightarrow A(x) \in G_{u(x)}$ for almost every $x \in \Omega$ is used, and, for each $x \in \Omega$, $G_{u(x)}$ is a set of fourth order tensors such that

$$
G_{u(x)}=\left\{\begin{array}{lll}
\left\{A_{0}\right\} & \text { if } & u(x)=0 \\
\left\{A_{1}\right\} & \text { if } & u(x)=1
\end{array}\right.
$$

Henceforth we choose, for all $x \in \Omega, G_{u(x)}$ as the set of all Hooke tensors obtained by homogenization of tensors $A_{0}$ and $A_{1}$ in proportion $1-u(x)$ and $u(x)$, respectively (see, e.g., [2] for details on homogenization). We recall in particular that $G_{u(x)}$ is closed. 
Proposition 7.1 The functional $\tilde{J}$ is continuous on $L^{\infty}(\Omega,[0,1])$ strongly in $L^{1}(\Omega)$.

Proof. Suppose that $\left(u_{n}\right) \in L^{\infty}(\Omega,[0,1])$ converges to $u \in L^{\infty}(\Omega,[0,1])$ strongly in $L^{1}(\Omega)$. Thus $u_{n} \rightarrow u$ almost everywhere for a non-relabeled subsequence. By compactness of the $G$ convergence (see Section 6) and stability of $G_{u_{n}}$ with respect to this convergence (see [2] Lemma 2.1.5), there exists $A_{n}^{*} \in G_{u_{n}}$ such that

$$
C\left(A_{n}^{*}\right)=\inf _{A \in G_{u_{n}}} C(A) .
$$

Using again the compactness of the $G$-convergence, there exists a subsequence such that $A_{n}^{*}$ $G$ - converges to some $A^{*}$, thus $C\left(A_{n}^{*}\right) \rightarrow C\left(A^{*}\right)$. By [2] Lemma 2.1.7, there exists $c, \delta>0$ independent of $x$ such that

$$
d\left(G_{u_{n}(x)}, G_{u(x)}\right) \leqslant c\left|u_{n}(x)-u(x)\right|^{\delta}
$$

for every $x \in \Omega$, where $d$ denotes the Hausdorff distance between sets. Hence there exists $A_{n}^{\sharp} \in G_{u}$ such that $\left|A_{n}^{*}-A_{n}^{\sharp}\right| \leqslant c\left|u_{n}-u\right|^{\delta}$ almost everywhere. By the dominated convergence theorem we get $\left\|A_{n}^{*}-A_{n}^{\sharp}\right\|_{L^{1}(\Omega)} \rightarrow 0$. Once more by compactness of the $G$-convergence, $A_{n}^{\sharp} G$-converges to some $A^{\sharp} \in G_{u}$, up to a subsequence. It follows from [2] Proposition 1.3.44 that $A^{*}=A^{\sharp} \in G_{u}$.

Let now $A \in G_{u}$ be arbitrary, and denote by $A_{n}(x)$ the projection of $A(x)$ onto $G_{u_{n}(x)}$. Using again (7.5), we get $A_{n}(x) \rightarrow A(x)$ almost everywhere, therefore, by [2] Lemma 1.2.22, $C\left(A_{n}\right) \rightarrow$ $C(A)$. By definition we have $C\left(A_{n}\right) \geqslant C\left(A_{n}^{*}\right)$ for all $n$. Passing to the limit yields $C(A) \geqslant C\left(A^{*}\right)$. This means that

$$
C\left(A^{*}\right)=\inf _{A \in G_{u}} C(A) .
$$

Eventually we have obtained

$$
\tilde{J}\left(u_{n}\right)=C\left(A_{n}^{*}\right)+\ell \int_{\Omega} u_{n} d x \rightarrow C\left(A^{*}\right)+\ell \int_{\Omega} u d x=\tilde{J}(u) .
$$

For $\varepsilon>0$ fixed we solve the approximate problem

$$
\inf _{u \in L^{\infty}(\Omega,[0,1])}\left\{\tilde{J}(u)+\alpha \tilde{F}_{\varepsilon}(u)\right\} .
$$

Using (2.3), (7.4) and (7.2), this can be rewritten as

$$
\begin{aligned}
\inf _{\substack{u \in L^{\infty}(\Omega,[0,1]), A \in G_{u} \\
(v, \sigma) \in H^{1}(\Omega) \times \Sigma}}\left\{\int_{\Omega} A^{-1} \sigma:\right. & \sigma d x+\ell \int_{\Omega} u d x \\
& \left.+\alpha\left[\frac{\varepsilon}{2}\|\nabla v\|_{L^{2}(\Omega)}^{2}+\frac{1}{2 \varepsilon}\left(\|v\|_{L^{2}(\Omega)}^{2}+\langle u, 1-2 v\rangle\right)\right]\right\} .
\end{aligned}
$$

Proposition 7.2 The infima (7.6) and (7.7) are attained. 
Proof. First, we remark that both problems (7.6) and (7.7) amount to solving

$$
\inf _{u \in L^{\infty}(\Omega,[0,1]), A \in G_{u}}\left\{E_{\varepsilon}(u, A):=C(A)+\ell \int_{\Omega} u d x+\alpha \tilde{F}_{\varepsilon}(u)\right\} .
$$

Let $\left(u_{n}, A_{n}\right)$ be a minimizing sequence. Thanks to the density of $L^{\infty}(\Omega,\{0,1\})$ in $L^{\infty}(\Omega,[0,1])$ for the weak-* topology of $L^{\infty}(\Omega)$ and the continuity of $E_{\varepsilon}(., A)$ for the same topology, see Lemma 2.3, we may assume (after a classical diagonalization procedure) that $\left(u_{n}\right) \in L^{\infty}(\Omega,\{0,1\})$. We extract a subsequence, still denoted $\left(u_{n}\right)$, such that $u_{n} \rightarrow u \in L^{\infty}(\Omega,[0,1])$ weakly-* in $L^{\infty}(\Omega)$. Further, by compactness of the $G$ convergence, we can extract a subsequence such that $\left(A_{n}\right) G$-converges to some tensor field $A$. By construction, we have $A \in G_{u}$. By definition of the $G$-convergence, the sequence of the states $\left(y_{n}\right)$, solutions of (7.1) with Hooke's tensor $A_{n}$, converges weakly in $H_{0}^{1}(\Omega)$ to the state $y$ associated to $A$. This implies that $C\left(A_{n}\right) \rightarrow C(A)$, and subsequently, using again Lemma 2.3, that $E_{\varepsilon}\left(u_{n}, A_{n}\right) \rightarrow E_{\varepsilon}(u, A)$.

\subsection{Description of the algorithm}

We use again an alternating minimization algorithm, by performing successively a full minimization with respect to each of the variables $\sigma, v,(u, A)$. The minimization with respect to $\sigma$ is equivalent to solving the linear elasticity problem (7.1) and setting $\sigma=A \nabla^{s} y$. The minimization with respect to $v$ is again done by solving (2.4). The minimization with respect to $A$ for a given $u$ reduces to the standard problem

$$
\inf _{A \in G_{u}}\left\{\int_{\Omega} A^{-1} \sigma: \sigma d x\right\}=: f(u, \sigma) .
$$

When $A_{1}$ and $A_{0}$ are isotropic and $A_{0} \rightarrow 0$, the minimization is achieved by using well-known lamination formulas, see [2]. We have

$$
f(u, \sigma)=A_{1}^{-1} \sigma: \sigma+\frac{1-u}{u} f^{*}(\sigma),
$$

with, in dimension $N=2$,

$$
f^{*}(\sigma)=\frac{2 \mu+\lambda}{4 \mu(\mu+\lambda)}\left(\left|\sigma_{1}\right|+\left|\sigma_{2}\right|\right)^{2} .
$$

Above, $\lambda, \mu$ are the Lamé coefficients of the phase $A_{1}$, and $\sigma_{1}, \sigma_{2}$ are the principal stresses. Let us finally focus on the minimization with respect to $u$. We have to solve

$$
\inf _{u \in L^{\infty}(\Omega,[0,1])}\left\{\int_{\Omega} \Phi_{\varepsilon, v, \sigma}(u(x)) d x\right\}, \quad \text { with } \Phi_{\varepsilon, v, \sigma}(u)=f(u, \sigma)+\ell u+\frac{\alpha}{2 \varepsilon} u(1-2 v) .
$$

This means that, at every point $x \in \Omega$, we have to minimize the function $s \in[0,1] \mapsto \Phi_{\varepsilon, v, \sigma}(s)$. From

$$
\Phi_{\varepsilon, v, \sigma}(s)=A_{1}^{-1} \sigma: \sigma+\frac{1-s}{s} f^{*}(\sigma)+\left[\ell+\frac{\alpha}{2 \varepsilon}(1-2 v)\right] s
$$

we obtain the minimizer

$$
u= \begin{cases}1 & \text { if } \ell+\frac{\alpha}{2 \varepsilon}(1-2 v) \leqslant 0, \\ \min \left(1, \sqrt{\left.\frac{f^{*}(\sigma)}{\ell+\frac{\alpha}{2 \varepsilon}(1-2 v)}\right)}\right. & \text { if } \ell+\frac{\alpha}{2 \varepsilon}(1-2 v)>0 .\end{cases}
$$


The stopping criteria for the inner and outer loops are the same as in Section 6, with $\Delta_{\max }=10^{-4}$.

\subsection{Numerical examples}

We first consider the classical cantilever problem, where $\Omega$ is a rectangle of size $2 \times 1$. The left edge is clamped, and a unitary pointwise vertical force is applied at the middle of the right edge. We choose the Lagrange multiplier $\ell=100$, and use a mesh containing 160601 nodes. Our findings are displayed in Figure 8.

Next we address the bridge problem, where $\Omega$ is a rectangle of size $2 \times 1.2$. The structure is clamped on two segments of lengths 0.1 located at the tips of the bottom edge, and submitted to a unitary pointwise vertical force exerted at the middle of the bottom edge. The chosen Lagrange multiplier is $\ell=30$, and the mesh contains 123393 nodes. Our results are depicted in Figure 9.

\section{Conclusion}

We have introduced a new approximate perimeter functional which fulfills all the mathematical properties needed to get the convergence of (sub)sequences of minimizers in a general setting. As it is gradient-free, this functional can be directly applied to characteristic functions. But, since it automatically penalizes the intermediate densities at convergence, it can also be combined with convexification or homogenization methods. The fact that it can be defined through the solution of a linear partial differential equation makes it particularly well-suited to the framework of topology optimization.

Acknowledgements. We thank M. Ciligot-Travain for useful remarks.
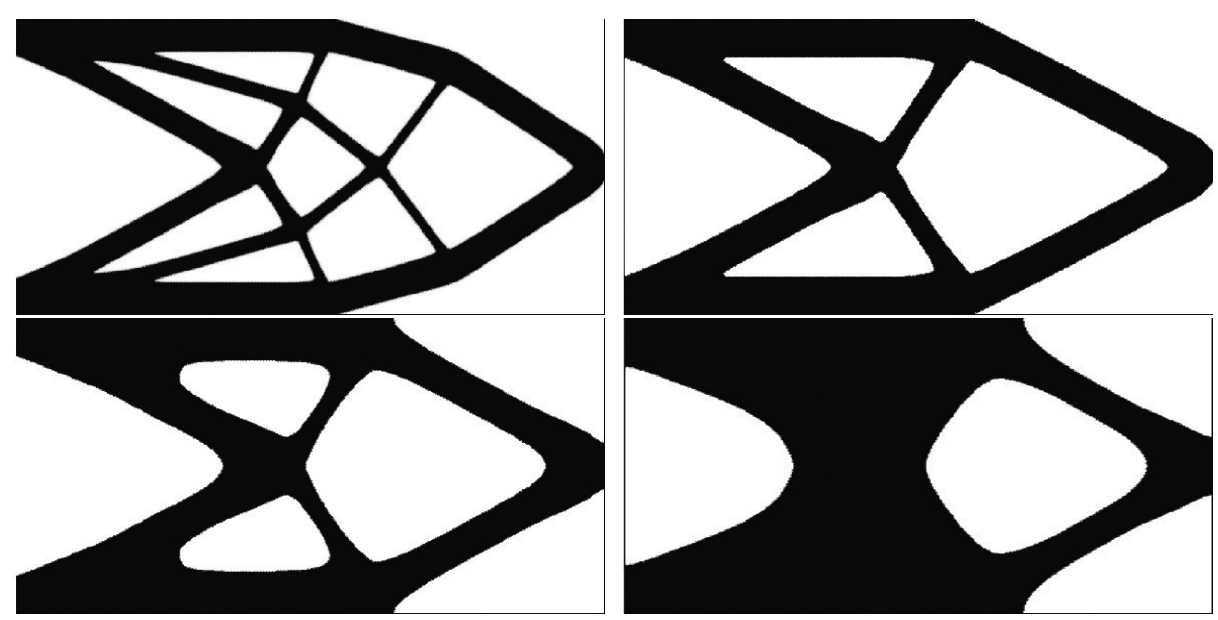

FIG. 8. Cantilever for $\alpha=0.1,2,20,50$, respectively 


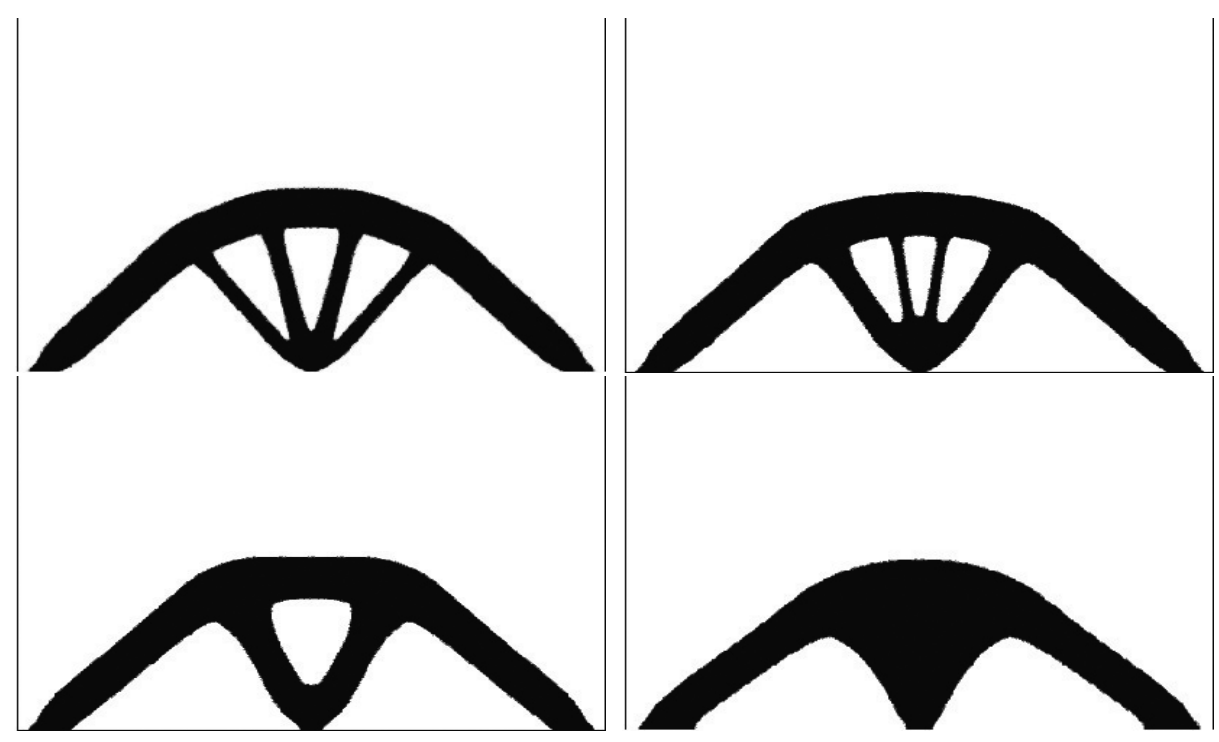

FIG. 9. Bridge for $\alpha=0.2,1,3,10$, respectively

\section{REFERENCES}

1. Alberti, G., \& Bellettini, G., A non-local anisotropic model for phase transitions: asymptotic behaviour of rescaled energies. European J. Appl. Math. 9 (1998), 261-284. Zb10932. 49018 MR1634336

2. Allaire, G., Shape optimization by the homogenization method, Applied Mathematical Sciences, vol. 146, Springer-Verlag, New York, 2002. Zb10990.35001 MR1859696

3. Allaire, G., Conception optimale de structures, Mathématiques \& Applications (Berlin) [Mathematics \& Applications], vol. 58, Springer-Verlag, Berlin, 2007, With the collaboration of Marc Schoenauer (INRIA) in the writing of Chapter 8. Zb11132.49033 MR2270119

4. Allaire, G., Bonnetier, E., Francfort, G., \& Jouve, F., Shape optimization by the homogenization method. Numer. Math. 76 (1997), 27-68. Zb10889.73051 MR1438681

5. Allaire, G., Jouve, F., \& Van Goethem, N., Damage and fracture evolution in brittle materials by shape optimization methods. J. Comput. Phys. 230 (2011), 5010-5044. Zblpre05920273 MR2795994

6. Ambrosio, L., \& Buttazzo, G., An optimal design problem with perimeter penalization. Calc. Var. Partial Differential Equations 1 (1993), 55-69. Zb10794. 49040 MR1261717

7. Ambrosio, L., Fusco, N., \& Pallara, D., Functions of bounded variation and free discontinuity problems. Oxford Mathematical Monographs, The Clarendon Press Oxford University Press, New York, 2000. Zb10957. 49001 MR1857292

8. Ambrosio, L., \& Tortorelli, V. M., Approximation of functionals depending on jumps by elliptic functionals via $\Gamma$-convergence. Comm. Pure Appl. Math. 43 (1990), 999-1036. Zb10722. 49020 MR1075076

9. Amstutz, S., Sensitivity analysis with respect to a local perturbation of the material property. Asymptot. Anal. 49 (2006), 87-108. Zbl1187.49036 MR2260558

10. AmstutZ, S., Regularized perimeter for topology optimization, Tech. report, HAL-00542854, 2010.

11. Amstutz, S., Connections between topological sensitivity analysis and material interpolation schemes in topology optimization. Struct. Multidiscip. Optim. 43 (2011), 755-765. MR2806146 
12. Amstutz, S., Novotny, A., \& Van Goethem, N., Minimal partitions and image classification using a gradient-free perimeter approximation. Submitted (available as preprint: hal-00690011, version 1, 20 Apr. 2012) (2012).

13. Attouch, H., Buttazzo, G., \& Michaille, G., Variational analysis in Sobolev and BV spaces. MPS/SIAM Series on Optimization, vol. 6, Society for Industrial and Applied Mathematics (SIAM), Philadelphia, PA, 2006, Applications to PDEs and optimization. Zbl1095.49001 MR2192832

14. Aujol, J.-F., \& Aubert, G., Optimal partitions, regularized solutions, and application to image classification. Applicable Analysis 84 (2005), 15-35. Zb11207.94004 MR2113653

15. Bendsøe, M. P., \& Sigmund, O., Topology optimization. Springer-Verlag, Berlin, 2003, Theory, methods and applications. MR2008524

16. Bourdin, B., \& Chambolle, A., Design-dependent loads in topology optimization. ESAIM, Control Optim. Calc. Var. 9 (2003), 19-48 (English). Zbl1066. 49029 MR1957089

17. Bourdin, B., Francfort, G. A., \& MARigo, J.-J., The variational approach to fracture. J. Elasticity 91 (2008), 5-148. Zbl1176.74018 MR2390547

18. BRAides, A., $\Gamma$-convergence for beginners. Oxford Lecture Series in Mathematics and its Applications, vol. 22, Oxford University Press, Oxford, 2002. Zb11198.49001 MR1968440

19. Chambolle, A., Cremers, D., \& Pock, T., A convex approach to minimal partitions. hal-00630947, version 1 (11 Oct. 2011).

20. Dal Maso, G., An introduction to $\Gamma$-convergence. Progress in Nonlinear Differential Equations and their Applications, 8, Birkhäuser Boston Inc., Boston, MA, 1993. Zb10816.49001 MR1201152

21. Fried, E., \& Gurtin, M. E., Continuum theory of thermally induced phase transitions based on an order parameter. Phys. D 68 (1993), 326-343. Zb10793. 35049 MR1242744

22. Fried, E., \& Gurtin, M. E., Dynamic solid-solid transitions with phase characterized by an order parameter. Phys. D 72 (1994), 287-308. Zb10812.35164 MR1271571

23. Garreau, S., Guillaume, P., \& Masmoudi, M., The topological asymptotic for PDE systems: the elasticity case. SIAM J. Control Optim. 39 (2001), 1756-1778 (electronic). Zb10990 . 49028 MR1825864

24. Gurtin, M. E., On a theory of phase transitions with interfacial energy. Arch. Rational Mech. Anal. 87 (1985), 187-212. MR0768066

25. Henrot, A., \& Pierre, M., Variation et optimisation de formes. Mathématiques \& Applications (Berlin) [Mathematics \& Applications], vol. 48. (2005) Zb11098.49001 MR2512810

26. Kawohl, B., Rearrangements and convexity of level sets in PDE. Lecture Notes in Mathematics, vol. 1150, Springer-Verlag, Berlin, 1985. Zb10593.35002 MR0810619

27. ModicA, L., The gradient theory of phase transitions and the minimal interface criterion. Arch. Rational Mech. Anal. 98 (1987), 123-142. Zbl0616.76004 MR0866718

28. Modica, L., \& Mortola, S., Il limite nella $\Gamma$-convergenza di una famiglia di funzionali ellittici. Boll. Unione Mat. Ital., V. Ser. A 14 (1977), 526-529 (Italian). Zb10364. 49006 MR0473971

29. Modica, L., \& Mortola, S., Un esempio di $\Gamma$-convergenza. Boll. Unione Mat. Ital., V. Ser. B 14 (1977), 285-299 (Italian). Zbl0356.49008 MR0445362

30. Rogers, R. C., \& Truskinovsky, L., Discretization and hysteresis. Physica B 233 (1997), 370-375.

31. SoкоŁowski, J., \& Żochowski, A., On the topological derivative in shape optimization. SIAM J. Control Optim. 37 (1999), 1251-1272 (electronic). Zb10940.49026 MR1691940

32. Solci, M., \& Vitali, E., Variational models for phase separation. Interfaces Free Bound. 5 (2003), 27-46. Zbl1041.49017 MR1959767

33. Van Goethem, N., \& Novotny, A., Crack nucleation sensitivity analysis. Math. Meth. Appl. Sc. 33 (2010), 1978-1994. Zbl1201. 35081 MR2744615

34. Willem, M., Principles of functional analysis. (Principes d'analyse fonctionnelle.) Nouvelle Bibliothèque Mathématique 9. Paris: Cassini., 2007 (French). Zb11205. 46001 MR2567317 